\title{
Development of stochastic isogeometric analysis (SIGA) method for uncertainty in shape
}

\author{
Hongguan Zhang ${ }^{1 *}, \quad$ Tadahiro Shibutani ${ }^{1^{*}}$ \\ ${ }^{1}$ Graduate School of Environment and Information Sciences, Yokohama National University, 79-5 \\ Tokiwadai, Hodogaya-ku, Yokohama, Kanagawa 240-8501, Japan
}

\begin{abstract}
SUMMARY
In this paper, a new method is proposed that extend the classical deterministic isogeometric analysis (IGA) into a probabilistic analytical framework in order to evaluate the uncertainty in shape, and aim to investigate a possible extension of IGA in the field of computational stochastic mechanics. Stochastic isogeometric analysis (SIGA) method for uncertainty in shape is developed by employing the geometric characteristics of the non-uniform rational basis spline (NURBS) and the probability characteristics of polynomial chaos expansions (PCE). The proposed method can accurately and freely evaluate problems of uncertainty in shape caused by deformation of the structural model. Additionally, we use the intrusive formulation approach to incorporate PCE into the IGA framework, and the $\mathrm{C}++$ programming language to implement this analysis procedure. To verify the validity and applicability of the proposed method, two numerical examples are presented. The validity and accuracy of the results are assessed by comparing them to the results obtained by Monte Carlo simulation (MCS) based on the IGA algorithm.
\end{abstract}

KEY WORDS: stochastic isogeometric analysis, uncertainty in shape, isogeometric analysis, polynomial chaos expansions.

In reality, various uncertainties exist in structural systems, because various the physical characteristics are not deterministic in the actual engineering application. Such characteristics include the material properties, boundary conditions and structural shape, etc. These uncertain

*Correspondence to:

Hongguan Zhang, E-mail: hongguan-zhang-np@ynu.jp;

Tadahiro Shibutani, E-mail: shibutani-tadahiro-bj@ynu.ac.jp;

Graduate School of Environment and Information Sciences, Yokohama National University, 79-5

Tokiwadai, Hodogaya-ku, Yokohama, Kanagawa 240-8501, Japan 
factors can be investigated by a numerical analysis method, that is commonly known as the uncertainty analysis method. In recent years, with the rapid development of risk-based rules and structure design methods, traditional deterministic numerical analysis methods have been unable to meet the need of analysing the uncertainty and randomness in practical engineering projects. Therefore, the development of uncertainty analysis methods is currently attracting an increasing amount of attention.

Generally, the stochastic finite element method (SFEM) is employed in order to analyse uncertain problems in the structural systems. The SFEM is an extension of the classical finite element method (FEM) that is from a deterministic numerical analysis framework to a stochastic framework. The SFEM has been widely applied in science and engineering as an important uncertainty analysis method. Initially, Astill and Shinozuka [1] have presented the Monte Carlo simulation method (MCS), which is a combination of the FEM and MCS. The latter is the most general and simplest approach for dealing with response variability in the structural system [2]. However, the MCS requires excessive computational power in comparison to other stochastic FEMs, and especially for handling complex models involving several stochastic variables. The perturbation method [3] overcomes this drawback, when the perturbation of response variability is in the first and second-order. The perturbation method was developed by applying the Taylor series expansion of the response vector into the physical system, and the results of the analysis are the distribution-free [4]. This method is limited within a minute perturbation range that is usually less than 20 or 30 percent of a variable's mean value. In recent years, another important branch of the SFEM has been presented by Ghanem and Spanos [5], namely, the spectral stochastic finite element method (SSFEM). in general, this method makes use of the Karhunen-Loève (K-L) expansion of the Gaussian random field in order to represent the uncertain parameters of a problem (such as material properties, nodal displacement, etc.). For the representation of nodal displacement in the SSFEM, an alternative approach has been provided and consists of using polynomial chaos expansions (PCE) [6]. Additionally, the PCE has also been applied to other SFEM. For instance, Honda [7] proposed spectral stochastic boundary element method (SSBEM) based on PCE and the K-L expansion in order to analyse the problem of uncertainty in shape in the boundary. Chen et al. [8] presented a new method of structural analysis for the solution of response uncertainty problems in the cases involving uncertainty in shape. The proposed method includes a mathematical formulation, which is a natural extension of the deterministic finite element concept to the space of random functions by the Hermite polynomial chaos expansion, in order to represent the uncertainty of shapes and the response surface. Developments have been described in some key articles [8-10].

Recently, isogeometric analysis (IGA) method was proposed by Hughes et al. [11] as an important alternative technology in computational mechanics. Its core idea was the use of the smooth geometric basis in CAD as the basis functions for numerical analysis [12]. This method successfully integrated computer aided design (CAD) and computer aided engineering (CAE) into a unified process, i.e. parameterizing the CAD objects to obtain an effective computation domain and generate a mesh, which is applied to the FEM analysis framework [13]. Xu et al. have done a lot of important work to improve the quality of the parameterization of the computational domain of CAD objects [14,15]. IGA extended rapidly to other fields of numerical analysis, including uncertainty analysis. Rossana et al. presented an innovative 
numerical method for computing the stress concentration factors in an isotropic plate with discontinuities by using IGA and SFEM [16]. Hien and Noh developed a perturbation technique in conjugation with IGA for the stochastic eigenvalue problem of free vibration of functionally graded material (FGM) plates with two random parameters for the elastic modulus and mass density, respectively [17]. Hien and Lam use IGA and MCS to address the bending of a plate under random load [18].

Actually, with the rapid development of industry and the improvement of structural design technology, the geometric shape of structures has become increasingly complex. Simultaneously, it has brought more new challenges to the field of uncertainty analysis techniques. Despite classical SFEM has excellent analytical performance and a sound analytical system, there are still some inevitable limitations. Especially, in the uncertainty analysis in shape, because of what its use of a geometry approximated by a finite element mesh (FE-mesh), some of its innate disadvantages have been exposed. In many situations, this geometry approximated can cause errors in the analytical results during the performing uncertainty analysis on some complex and sensitive geometric structures. In order to overcome the shortcomings in the SFEM mentioned above, based on the natural characteristics of NURBS, we proposed the stochastic isogeometric analysis method to deal with the problem of uncertainty in shape. The main contributions of this study are as follows:

- In this study, we proposed to introduce PCE into the IGA analysis framework to address the uncertainty problems in shape and given a formalization method.

- The numerical solving can be obtained without setting the coefficients of the degree of change at each node which are needed in the SFEM. Therefore, our work effectively improves computational efficiency and reduces the complexity of the analysis.

- From the perspective of probabilistic analysis, the random field is directly applied to an exact geometric entity through the control point, rather than an approximate mesh structure. Therefore, it is sensitive to geometric shape changes, which effectively reduced geometrical errors of numerical solving.

- Unlike the MCS analysis mechanism, the analytical framework of the proposed method is constructed using the intrusive method and is therefore very scalable, and the construction of stochastic response surface does not require multiple simulations, thus it can deliver very fast results at minimum computational cost.

- Moreover, by inheriting the advantage of IGA, the proposed SIGA method has several unique advantages compared to SFEM. For instance, using the smoother, highly continuous basis function, possessing good interactivity with $\mathrm{CAD}$, and the ability to exactly represent some commonly encountered geometry such as circles and ellipses, etc.

The remainder of this paper is structured as follows: In Sections 2 and 3, we present some of the basic concepts of IGA and probability theory associated with this study, respectively. The formulation of the proposed approach (SIGA) is presented in Section 4. Numerical examples are provided in Section 5. The future works and conclusions drawn from this study are presented in Section 6. 
2 Plenty of approaches have been used to structure the geometric model in CAD. In general, the non-uniform rational basis spline (NURBS) basis function has been used to build the geometric model, and has served as shape function in the pre/post processing of isogeometric analysis 5 [12].

\subsection{B-spline basis and knot vector}

In this section, a brief description of the B-spline is given. B-spline is very important for building the NURBS. The B-splines basis functions are formulated via the Cox-de Boor 9 recursion formulation [11], as follows:

$$
N_{i, p=0}(\xi)=\left\{\begin{array}{lr}
1 & \text { if } \xi_{i} \leq \xi<\xi_{i+1} \\
0 & \text { otherwise }
\end{array}\right.
$$

where $i$ is the knot index, i.e., $i=1,2, \cdots n+p+1 . p$ is the order of the $\mathrm{B}$-splines basis functions. $N_{i, p=0}(\xi)$ is the zeroth-order B-splines basis function and $N_{i, p}$ is the piecewise linear function. $\xi_{i}$ are knots of the non-descending knot vector in the parameter space, that can be mapped into the physical space, and define a physical mesh on the geometric entity by partitioning it into the elements. The corresponding knot vector is expressed by

$$
\Xi=\left\{\xi_{1}, \xi_{2}, \cdots, \xi_{n+p+1}\right\}
$$

For $p>0$, the basis-functions are defined by the following equation:

$$
N_{i, p}(\xi)=\frac{\xi-\xi_{i}}{\xi_{i+p}-\xi_{i}} N_{i, p-1}(\xi)+\frac{\xi_{i+p+1}-\xi}{\xi_{i+p+1}-\xi_{i+1}} N_{i+1, p-1}(\xi)
$$

The derivative of the $i$ th-order B-spline basis is expressed by

$$
\frac{d N_{i, p}(\xi)}{d \xi}=\frac{p N_{i, p-1}(\xi)}{\xi_{i+p}-\xi_{i}}-\frac{p N_{i+1, p-1}(\xi)}{\xi_{i+p+1}-\xi_{i+1}}
$$

\subsection{B-spline curves and B-spline surfaces}

In the $d$-dimensional space $\mathbb{R}^{d}$, the $p$ th-order B-spline curve $C(\xi)$ is built by

$$
C(\xi)=\sum_{i}^{n} N_{i, p}(\xi) B_{i}
$$

where $N_{i, p}(\xi)$ are the B-spline basis functions of order $p$ defined on the open knot vector, which is nonperiodic and non-uniform. $B_{i}$ represents the control points, which are the vectorvalued coefficients of the B-spline basis functions. An example piecewise quadratic B-spline curve in $\mathbb{R}^{2}$ is shown in Figure 1, where the control points are denoted by red solid circles. 
The dashed line connecting the control points is referred to as the control polygon or control net, which is a piecewise linear interpolation of the control points. In Figures 1(a) and 1(b), the two B-spline curves are built from the open knot vector, $\Xi=\{0,0,0,1,2,2,3,4,4,4\}$. Therefore, the curve is interpolatory at the first and last control points. Additionally, it is also interpolatory at the fourth control point, since the multiplicity of the knot $\xi=2$ is equal to the basis order. Note that the two B-spine curves are built by using the same basis function and order, with the only difference being that the coordinate of control point 2, i.e., the curve in Figure 1(b) is obtained by moving the control point 2 in Figure 1(a).

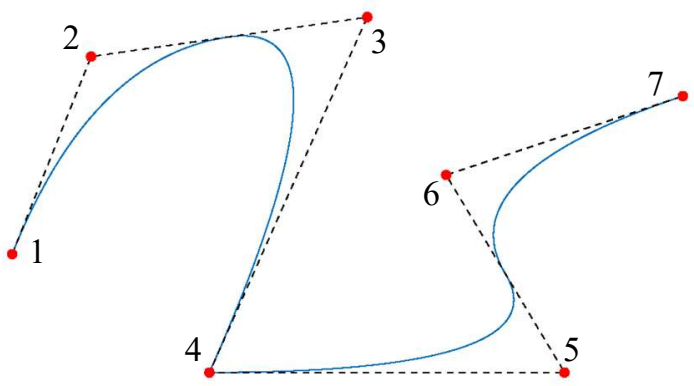

(a) Shape of curve before moving control point 2

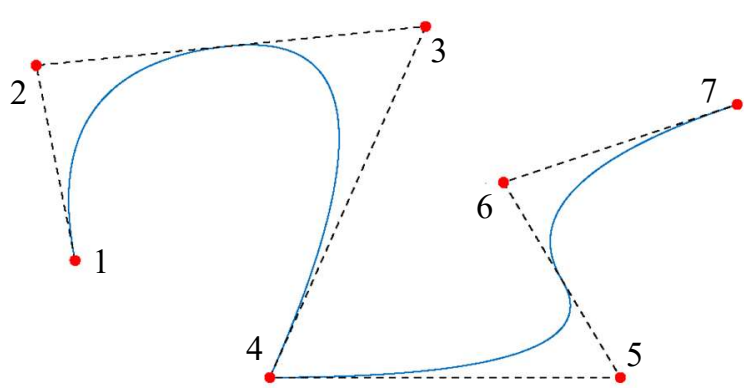

(b) Shape of curve after moving control point 2

Figure 1: Piecewise quadratic B-spline curve, control polygon, and control points in $\mathbb{R}^{2}$.

The B-spline surface is constructed by taking the control net $\left\{B_{i, j}\right\}, i=1,2, \cdots, n ; j=1,2, \cdots, m$, the knot vectors $\Xi$ and $H$, and the B-spline basis functions in the direction of the two knot vectors, as follows:

$$
S(\xi, \eta)=\sum_{i=1}^{n} \sum_{j=1}^{m} N_{i, p}(\xi) M_{j, q}(\eta) B_{i, j}
$$

with

$$
\begin{aligned}
& \Xi=\left\{\xi_{1}, \xi_{2}, \ldots, \xi_{n+p+1}\right\} \\
& \mathrm{H}=\left\{\eta_{1}, \eta_{2}, \ldots, \eta_{m+q+1}\right\}
\end{aligned}
$$

where $N_{i, p}(\xi)$ and $M_{j, q}(\eta)$ are the basis functions of order $p$ and $q$, respectively. An example of the B-spline surface is shown in Figure 2, where the mesh lie in the surfaces are defined by knots in the knot vectors $\Xi=\{0,0,0,0,1 / 7,2 / 7,3 / 7,4 / 7,5 / 7,6 / 7,1,1,1,1\}$ and $\mathrm{H}=\{0,0,0,1 / 8,2 / 8,3 / 8,4 / 8,5 / 8,6 / 8,7 / 8,1,1,1\}$, that partitioned the surfaces into the elements. The element boundaries in the surface are simply the images of knot lines under the geometric mapping. An initial surface is shown in Figure 2(a), that is plane since all the control points located in a common plane, and the corresponding control net is offset from the surface in order to obtain the better visualization. The surface in Figure 2(b) is the image after the surface in Figure 2(a) was changed, which was obtained by moving the control points B (marked in the red dot) in the control net. 
As described above, one of the important properties of B-splines was shown, that is its ability to directly change geometrical shape by adjusting the control points. For examples, geometry in Figures 1 and 2, by adjusting the control points, the geometric shape can be easily changed. Besides, note that due to the modification scheme property and local support property of Bsplines [19], the area that each control point can affect is local and controllable. As shown in Figure 2, if control point B is moved to a new location, it only can affect the shape of the partially adjacent area on the surface and elsewhere is unaffected. In this study, this property of the B-spline was fully utilised, and the structural analysis with consideration to the uncertainty in shape was implemented by importing the parameters of uncertainty (mean and deviation etc.) Into the control point coordinates.

12

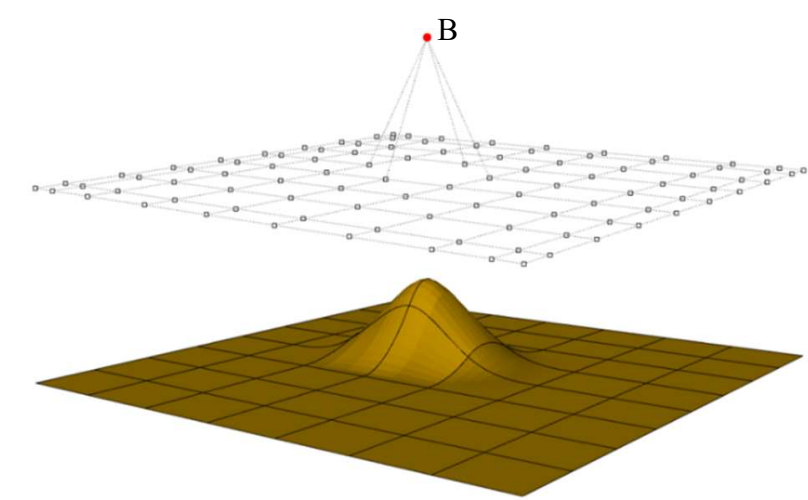

(b) Geometry after moving control point B

(a) Original geometry

Figure 2: The cubic $\times$ quadratic surface B-spline surfaces and control nets in $\mathbb{R}^{2}$.

\subsection{NURBS}

The NURBS is a more flexible modelling approach based on the B-spline concept, but without the drawbacks of B-spline; namely, NURBS allows exact the representation of geometrical shapes with conic sections, such as cylinders, ellipsoids, etc. However, it is impossible to use the B-spline to represent these simple shapes exactly. The NURBS basis function is defined as follows:

$$
R_{i, p}(\xi)=\frac{N_{i, p}(\xi) w_{i}}{\sum_{\hat{i}=1}^{n} N_{\hat{i}, p}(\xi) w_{\hat{i}}}
$$

where $R_{i, P}(\xi)$ represents the piecewise rational functions on the knot vector, and $w_{i}$ are the weights applied to an affine transformation of the B-spline curves between the highdimensional and low-dimensional spaces. Correspondingly, the derivatives of the NURBS basis are given by

$$
\frac{d R_{i, p}(\xi)}{d \xi}=w_{i} \frac{W(\xi) N_{i, p}^{\prime}(\xi)-W^{\prime}(\xi) N_{i, p}(\xi)}{(W(\xi))^{2}}
$$

where 


$$
W^{\prime}(\xi)=\sum_{\hat{i}}^{n} N_{\hat{i}, p}^{\prime}(\xi) w_{\hat{i}}
$$

2 The NURBS curve and surface are defined by the rational basis functions and corresponding

3 control points, as follows

\section{STOCHASTIC REPRESENTATION FOR SIGA}

This section focuses on the stochastic analysis techniques employed in this study. The idea of SIGA with uncertainty in shape proceeds mainly as follows: (1) The inherent randomness of a system is considered as an input, which is defined by the probability density function (PDF) of the random variable. (2) IGA is used in conjunction with the PCE of the Gaussian random field describing the analysis process. (3) The responses of the system are obtained by assuming that is a function for inputting random variables. The role of the PCE in this study is very important, since it is used to represent uncertain parameters. Thus, the PCE properties employed in this study are discussed below.

\subsection{Representation of SIGA input}

Firstly, the representation of input within the framework of uncertainty analysis is given, which usually consists of inherent randomness of the structural system, such as, shape, loads, properties of materials etc. In this study, the uncertainty in shape was considered as a random variable. In addition, the PCE was used to represent this uncertain parameter and taken as an input parameter. When the input parameter followed a normal distribution, the input parameter $\mathrm{H}(\zeta)$ was defined as follows:

$$
\mathrm{H}(\zeta)=\mathrm{H}_{\mu}+\mathrm{H}_{\sigma} \zeta
$$

where $\mathrm{H}_{\mu}$ is the mean value of the input parameter. $\mathrm{H}_{\sigma}$ is the standard deviation for the inherent randomness of the system, and $\zeta$ is the standard normal probabilistic variable.

\subsection{Polynomial chaos expansion (PCE)}

In uncertainty analysis, the stochastic expansion method is a significant alternative approach for representing uncertain parameters [20]. The purpose of stochastic expansion is to better describe the uncertainty of the system by introducing a series of polynomials characterizing the characteristics of the stochastic system.

In this study, the PCE was employed in order to represent inherent uncertainty in structure model. PCE approach uses a random space composed of polynomial bases to describe the 
uncertainty of system with PDF form. The basic idea is to approximately represent uncertainty by using the sum of the orthogonal polynomial chaos containing independent random variables, and the key step is to determine the coefficients of each polynomial. The PCE with multiple random variables for a Gaussian random response is defined as follows [21]:

$$
u(\zeta)=a_{0} \Psi_{0}+\sum_{i_{1}=1}^{\infty} a_{i_{1}} \Psi_{1}\left(\vartheta_{i_{1}}(\zeta)\right)+\sum_{i_{1}=1}^{\infty} \sum_{i_{2}=1}^{i_{1}} a_{i_{i_{2}}} \Psi_{2}\left(\vartheta_{i_{1}}(\zeta), \vartheta_{i_{2}}(\zeta)\right)
$$

$$
+\sum_{i_{1}=1}^{\infty} \sum_{i_{2}=1}^{i_{1}} \sum_{i_{3}=1}^{i_{2}} a_{i_{1} i_{3}} \Psi_{3}\left(\vartheta_{i_{1}}(\zeta), \vartheta_{i_{2}}(\zeta), \vartheta_{i_{3}}(\zeta)\right)+\cdots
$$

where $u(\zeta)$ is a random process that can express the input parameter and can approximate the response; $a_{i_{1}}, \cdots, a_{i_{p}}$ are polynomial coefficients; $\vartheta_{i_{1}}(\zeta), \ldots, \vartheta_{i_{p}}(\zeta)$ is a set of random variables in the sample space; $\Psi_{p}\left(\vartheta_{i_{1}}(\zeta), \ldots, \vartheta_{i_{p}}(\zeta)\right)$ is a set of orthogonal polynomials, which are functions of the random variable $\vartheta_{i_{p}}(\zeta)$.

Equation (14) can be reduced to:

$$
u(\zeta)=\sum_{i=0}^{q} a_{i} \Psi_{i}(\vec{\vartheta}(\zeta))
$$

where $\vec{\vartheta}(\zeta)$ is the random vector built from random variables. $q$ is the number of terms of polynomial chaos, and is determined by the following formula:

$$
q=\frac{(p+n) !}{p ! n !}-1
$$

where $p$ is the maximal order of polynomial chaos, and $n$ is the number of random variables. According to the Askey-scheme [22], there are different optimal polynomials for different probability density functions, i.e., the choice of polynomial depends on the probability density function of the random variable. In this study, the stochastic response $u(\zeta)$ was approximated by using the Hermite polynomials as optimal polynomials, because uncertainty in shape was hypothetically defined as following the normal random distribution. The multi-dimensional Hermite polynomials are expressed as follows:

$$
\Psi_{p}\left(\zeta_{i_{1}}, \cdots, \zeta_{i_{p}}\right)=e^{-\frac{1}{2} \xi^{T} \vec{\xi}}(-1)^{p} \frac{\partial^{p}}{\partial \zeta_{i_{1}} \cdots \partial \zeta_{i_{p}}} e^{\frac{1}{2} \vec{T}^{T} \xi}
$$

Uncertainty in shape was assumed to be the only random variable in this study. Thus, the singlevariable version of the Hermite polynomials is given by Equation (17) [20]

$$
\left\{\Psi_{p}(\zeta)\right\}=\left\{1, \zeta, \zeta^{2}-1, \zeta^{3}-3 \zeta, \zeta^{4}-6 \zeta^{2}+3, \zeta^{5}-10 \zeta^{3}+15 \zeta, \ldots\right\}
$$


Note that the basis-functions constituting PCE are all orthogonal polynomials. Therefore, we used the orthogonal property of polynomials to deal with stiffness equations with random variables in this study. The orthogonal property of the Hermite polynomials is defined by the inner product of the weight functions as follows:

$$
\left\langle\Psi_{m}(\zeta), \Psi_{k}(\zeta)\right\rangle=\int_{D} \Psi_{m}(\zeta) \Psi_{k}(\zeta) W(\zeta) d \zeta=k ! \delta_{k m}
$$

where $\delta_{k m}$ is the Kronecker delta and $D$ is the domain of the standard normal probabilistic variable, and $W(\zeta)$ is the weight function of the Hermite polynomials. Generally, the weight function is the same as the probability density function, in order to ensure that the PCE converges exponentially to a random variable. For the random variable whose probability density function is a Gauss function, the weight function is expressed as follows:

$$
W(\zeta)=\frac{1}{\sqrt{2 \pi}} e^{\frac{-\zeta^{2}}{2}}
$$

and $\Psi_{m}(\zeta)$ is derived by the following characteristic:

$$
\left\langle\Psi_{m}(\zeta)\right\rangle=\left\{\begin{array}{lr}
1 & m=0 \\
0 & \text { if } m>0
\end{array}\right.
$$

As mentioned above, if the response surface is obtained by the PCE; then, the mean value and standard deviation of the stochastic response can be approximated by the following formulas:

$$
\begin{gathered}
E[u]=\left\langle\sum_{j=0}^{P} u_{j} \Psi_{j}(\zeta)\right\rangle=u_{0}\left\langle\Psi_{0}(\zeta)\right\rangle+\sum_{j=1}^{P} u_{j}\left\langle\Psi_{j}(\zeta)\right\rangle=u_{0} \\
\operatorname{Var}[u]=\left\langle(u-E[u])^{2}\right\rangle=\left\langle\left(\sum_{j=0}^{P} u_{j} \Psi_{j}(\zeta)-u_{0}\right)^{2}\right\rangle=\sum_{j=1}^{P} u_{j}^{2}\left\langle\Psi_{j}(\zeta)^{2}\right\rangle
\end{gathered}
$$

Furthermore, the probability density function can also be obtained by the output responses. Thus far, some important aspects of PCE have been described with respect to uncertainty analysis. These properties will be used in the formulation presented in the next chapter.

\section{FORMULATION OF STOCHASTIC ISOGEOMETRIC ANALYSIS FOR} UNCERTAINTY IN SHAPE

In this study, we combined IGA and the stochastic methodology in order to create a new method for estimating the uncertainty in shape. Moreover, in this section, we use two terminologies ("physically-based" and "FEA-based") in order to describe the different analytic viewpoint between the proposed method and the SFEM. These two terminologies have been used in earlier methods integrating FEM and computational geometry [12]. In this study, 
uncertainty in shape was considered from a physically-based point of view, instead of the classic FEA-based point of view. The following sections briefly discuss the application of these two analytic points of view within uncertainty analysis framework, respectively. Subsequently, the formulation of SIGA for uncertainty in shape will be defined.

\subsection{Classic FEA-based point of view for uncertainty analysis}

In general, the use of traditional SFEM to discuss uncertainty in shape is based on the FEAbased point of view, i.e. the SFEM is built from the analytical framework of the classic FEM [8-10]. Thus, it inherits all the characteristics of FEA in the term of geometry and algebra. It is regrettable that the some of the characteristics inherited may hinder the further development of SFEM in terms of uncertainty in shape, although they may not have any impact on the numerical analysis in the FEM or other SFEM. Among them, a major development bottleneck is the geometric representation of the analysis object. The requirements for the performance of the geometric shapes are usually relatively high in analyzing uncertainty in shape, especially for complex geometric structures. However, throughout the probability analysis process, SFEM use a geometry approximated by FE-mesh to represent the uncertainty in shape, namely, the random field is actually acted upon an approximate geometry instead of an actual geometric structure, so that leads to geometric errors, inevitably. Notwithstanding, through some mesh refinement methods inherited from the FEM, the analytical object can be as much as possible conform to the actual geometric structure, but this typical geometric error cannot be reduced indefinitely. Moreover, the analytical model is partitioned into the elements connected by nodes, and through linear interpolation shape functions to achieve interpolation within the elements. Therefore, all the information is tied to the finite element mesh, in the SFEM, the uncertain parameter information is introduced into nodal coordinates as well, in order to represent the uncertainty in shape. As the position of the corresponding node changes, the shape of the mesh and elements also changes. Thus, in order to avoid affecting the accuracy of the analytical results, the appropriate FE-mesh must be restructured by remeshing, repeatedly. This increases the difficulty and time of analysis. Furthermore, the FE-mesh is usually insensitive to detecting shape changes at the geometrical boundaries containing surface or curve, or even failing to represent the deformation process. For instance, there is a plate with a circular hole and we assume that the random field is acted on the location of the circular hole. At present, within the SFEM analytical framework, the uncertainty at hole can only be considered as changes in the size of the radius [8]. If we want to assume that the uncertainty representation is the shape change from circular to other irregularities, which is difficult to be achieved in the current SFEM analytical framework, let alone more complex geometric structures. If it is in the case of FEM, the geometric shape can be artificially and arbitrarily changed by the geometric visualization tools, and then repartition it into the FE mesh. However, in the actual analysis and formulation, the shape of the analytical object cannot be controlled artificially and visually, instead, use the algebraic method to deal with the analysis process. Therefore, in the analysis process of SFEM, the uncertainty representation in shape cannot be achieved simply by changing the coordinates of nodes with uncertain parameters, and also need to consider its influence on other elements and nodes. This influence is difficult to be controlled and determined for complex uncertainty representation. In Reference [8-10], Chen et al. by setting 
the coefficients of the degree of change at each node to represent the uncertainty in shape and gave the corresponding algorithm, but that cannot perform well for geometric structures with curves and surfaces due to the drawbacks inherited from the FEM mentioned above. Besides, these coefficients of the degree of change also there is an impact on the analytical results to a certain degree. Namely, if the bad coefficients are determined, a bad FE-mesh will be generated, resulting in an inaccuracy result that may be obtained [23].

Through the discussion of the above classic FEA-based point of view, we can know that despite the powerful FEM has been widely expanded and applied to various engineering fields, and has achieved prominent achievements. However, for the uncertainty analysis in shape based on FEM analysis framework, some inherent characteristics of FEM limit its development and greatly increase the difficulty and cost of analysis. Therefore, in order to break these bottlenecks, in this study we introduced the physically-based analytical point of view to deal with the uncertainty in shape.

\subsection{Physically-based point of view for uncertainty analysis}

In this study, a novel method is proposed in the aspect of reliability analysis for uncertainty in shape, and it is carried out from a physically-based point of view, i.e. the entire probability analysis uses an actual geometric structure to represent uncertainty in shape instead of an approximate one. Similarly, the SIGA for uncertainty in shape is an extension from the classical isogeometric analysis framework. Some of the characteristics inherited from IGA make up for the shortcomings of traditional SFEM on the uncertainty analysis in shape. The SIGA takes full advantage of these the native characteristics of IGA, that is using the same geometry description in engineering design and analysis processes, respectively [12]; Thus the random field is acted upon the geometric entity, the geometric errors are eliminated to the utmost, especially, the more complex the geometry, the more obvious this advantage, for example, circle and ellipse, and irregular geometry, etc. And, since a physical mesh on the geometric entity is used for discretization in the uncertainty analysis process, it does not need to be like FEM the mesh repartition due to shape change and determining coefficients of the degree of shape at each node, so the calculation load is reduced.

Another major advantage of the physically-based point of view, besides being precision in representing the complex geometric structure and its less computationally expensive, is that it can define the analytical boundaries flexibly, exactly and easily. This advantage is obtained based on local support property and the local modification scheme property of NURBS [19], coupled with the power of NURBS which ability to directly manipulate the geometric shape by adjusting the control points. Therefore, the uncertainty in shape can be represented arbitrarily, and region and degree of shape change can be controlled easily, flexibly and exactly. The specific example shown in Figure 3 consists of one-quarter of a circular disk with a circular hole at the centre. It was assumed that the shape of the circular hole entails uncertainty. As shown in Figure 3(a), the control points 1,2, and 3 control the shape of the hole directly, and any one of them can import uncertain parameters to represent the uncertainty in shape at the circular hole. Therefore, the determination and control of the analytical boundary for the region of interest became dramatically easier. We do not need to consider moving other control points to represent uncertainty in shape and the impact on the ones. 
Through the above discussion, it is obvious that the natural geometric features of NURBS provides great convenience for uncertainty analysis in shape. Here, it should be mentioned again that the ability of control points manipulating geometric shape intuitively and freely was adequately exploited to achieve representation of uncertainty in shape in this study. Thereby, degrees of freedom and uncertain parameters are located at the control points. And based on an affine invariance property that follows from the partition of unity property in the NURBS $[12,19]$, ensures that the random field acts precisely on the geometric entity through the control points, in spite of the fact that the control points are commonly not interpolatory in NURBS, unlike in standard FEM. Let us look at some details from the algebraic perspective. For better illustrative on this point, we suppose a two-dimensional geometry was structured from the Equation (12), and the let control point $B_{k, h}$ be introduced uncertainty, i.e. the control point $B_{k, h}$ was moved to a new position $B_{k, h}+\vec{v}$, where $\vec{v}$ is randomly varying geometric vector used to represent uncertainty in shape. Since the new NURBS geometric structure was defined by $B_{1,1}, \cdots, B_{k, h}+\vec{v}, \cdots, B_{n, m}$, as follows:

$$
\begin{aligned}
S^{\prime}(\xi, \eta) & =\sum_{i=1}^{k-1} \sum_{j=1}^{h-1} R_{i, p}(\xi) R_{j, q}(\eta) B_{i, j}+R_{k, p}(\xi) R_{h, q}(\eta)\left(B_{k, h}+\vec{v}\right)+\sum_{i=k+1}^{n} \sum_{j=h+1}^{m} R_{i, p}(\xi) R_{j, q}(\eta) B_{i, j} \\
& =\sum_{i=1}^{n} \sum_{j=1}^{m} R_{i, p}(\xi) R_{j, q}(\eta) B_{i, j}+R_{k, p}(\xi) R_{h, q}(\eta)\left(B_{k, h}+\vec{v}\right) \\
& =S(\xi, \eta)+R_{k, p}(\xi) R_{h, q}(\eta) \vec{v}
\end{aligned}
$$

It can be clearly known from Equation (24), only the $k, h$-th term used a control point containing uncertainty, $B_{k, h}+\vec{v}$, after regrouping, the new geometric structure is the sum of the original one and a $R_{k, p}(\xi) R_{h, q}(\eta) \vec{v}$. Furthermore, the randomly varying geometric vector $\vec{v}$ can be expressed by coordinates of the movement of control point, $\vec{v}=(\hat{x}-x, \hat{y}-y)$, where $x$ and $y$ are the control point coordinates before the shape change. $\hat{x}$ and $\hat{y}$ are the coordinates after the shape change. And its representation form regard with uncertainty can specifically be given by coordinate of the corresponding control points and a linear function with a random variable, $\zeta$, as follows:

$$
\hat{x}=x+L_{x}=x+\sum_{i=0}^{n} L_{x_{i}} \Psi(\zeta)
$$

$$
\hat{y}=y+L_{y}=y+\sum_{i=0}^{n} L_{y_{i}} \Psi(\zeta)
$$

where $\Psi(\zeta)$ is the polynomial (Hermite polynomials) chaos with random variables. $L_{x}$ and $L_{y}$ are the deviation lengths in the $x$ - and $y$-direction, respectively. In this study, the representation of uncertainty in shape is assumed to follow a normal distribution, such that the deviation lengths $\left(L_{x}\right.$ and $\left.L_{y}\right)$ can be rewritten as: 


$$
L_{y}=\sum_{i=0}^{n} L_{y_{i}} \Psi(\zeta)=L_{y}^{\mu}+L_{y}^{\sigma} \zeta
$$

where $L_{x o r y}^{\mu}$ and $L_{x o r y}^{\sigma}$ are the mean and standard deviation, respectively, the subscripts $x$ and $y$ represent the $x$ - and $y$-direction in the coordinate space. Figure 3(b) shows an example of uncertainty representation with regard to geometrical shape. We assumed that the deformation of structure model occurs along the inner circular hole. In this example, the uncertainty in shape of the model was represented by introducing the uncertain parameter into control point 1 , which is denoted by the hollow circle. Control point $\hat{1}$ (red solid node) is the location of control point 1 after shape changed, and the bidirectional arrow (in black) indicates the deviation length $(L)$. As shown in Figure 3(b), we can see that the shape of the inner circular hole can be changed simply by moving control point 1 , and without having to move other points. Additionally, the deviation length $(L)$ is introduced directly into the coordinate of the control point in order to geometrically represent the uncertainty in shape.

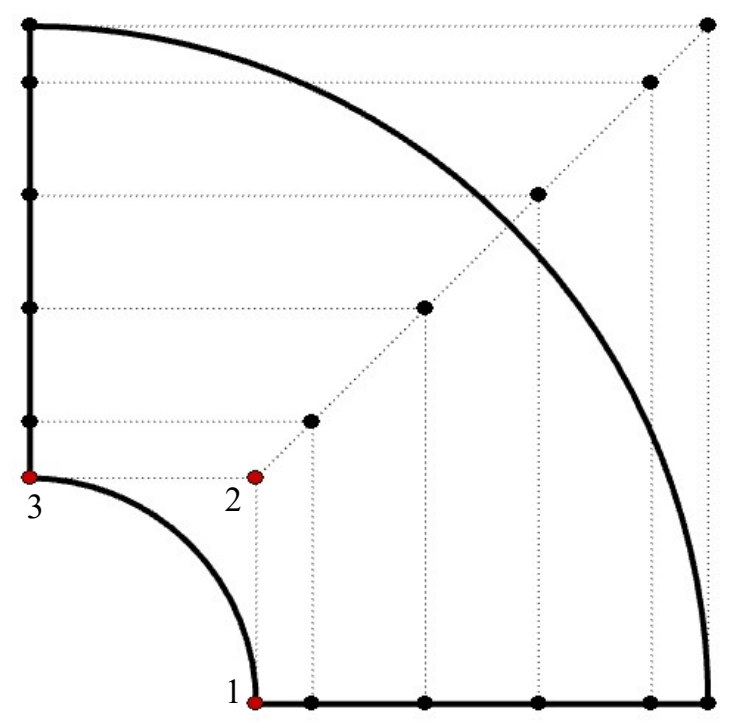

(a) Control points 1,2 and 3 for controlling circular hole at the centre.

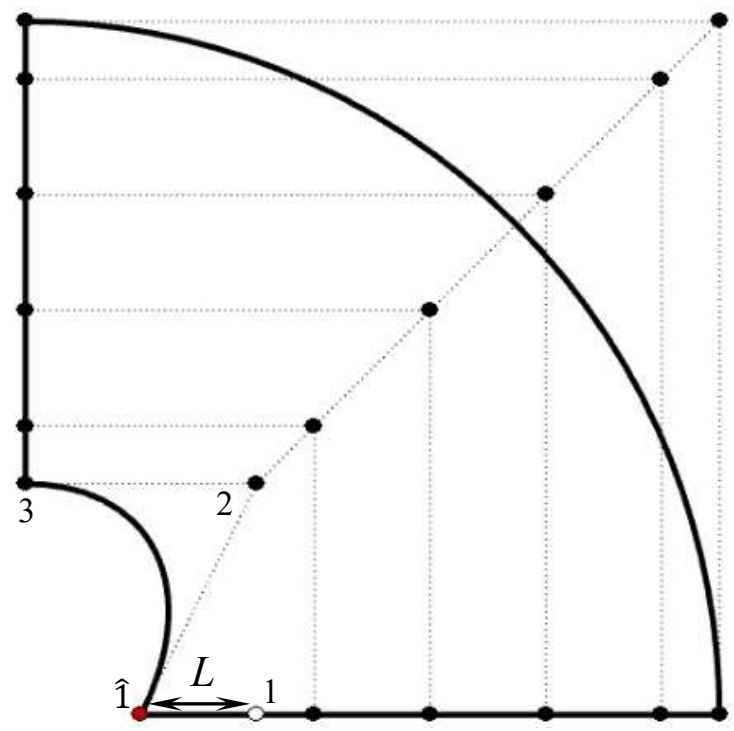

(b) Shape of circular hole at centre is changed by moving control point 1 to $\hat{1}$.

Figure 3: One quarter of circular disk with circular hole at centre

\subsection{Incorporating PCE into IGA Formulation}

In this study, the SIGA framework was developed by utilising the intrusive method. The formulation of SIGA was defined by incorporating the PCE into the original IGA formulation framework. Therefore, Stiffness equation in original IGA formulation was redefined in the form of PCE. In this section, the formulation of SIGA is given with respect to a twodimensional linear elasticity problem with a single random variable. 
Firstly, an abstract weak form is given by using the Galerkin's method in the finitedimensional subspaces of the trail solution space $S$ and by weighting function space $W$ [12,24], these subspaces are denoted by $S^{h}$ and $W^{h}$, respectively. The Galerkin approximation of the weak formulation is as follows: given body-force $f: \Omega \rightarrow \mathbb{R}$ and boundary conditions $r: \Gamma \rightarrow \mathbb{R}$, find $u^{h} \in S^{h}$,such that for all $w^{h} \in W^{h}$

$$
a\left(w^{h}, u^{h}\right)=\left(w^{h}, f\right)+\left(w^{h}, r\right)_{\Gamma}
$$

where the $a(\bullet, \bullet)$ and $(\bullet, \bullet)$ denote the bilinear form and inner-product form, respectively (see [11] and [24] for more details). In this study, the finite-dimensional subspaces $S^{h}$ and $W^{h}$ are constructed from NURBS. Therefore, $u^{h}$ and $w^{h}$ can be written as

$$
\begin{aligned}
& u^{h}=\sum_{A=1}^{n} c_{A} R_{A} \\
& w^{h}=\sum_{B=1}^{n} d_{B} R_{B}
\end{aligned}
$$

where $c_{A}$ and $d_{B}$ are the control variables. Then, the matrix equation can be constructed as follows:

$$
\mathbf{K d}=\mathbf{F}
$$

where $\mathbf{K}$ is the global stiffness matrix, $\mathbf{F}$ is the global force vector, and $\mathbf{d}$ is the unknown displacement response in this formulation. The global analysis system is assembled from the local stiffness matrices $\boldsymbol{k}_{e}$ and force vectors $\boldsymbol{f}_{e}$ established over the elements $\Omega^{e}$.

In the SIGA formulation, the local stiffness matrix containing the random variables $\zeta$ is expressed as follows:

$$
\boldsymbol{k}_{e}(\zeta)=\int_{\Omega^{e}} \boldsymbol{B}^{T}(\zeta) \boldsymbol{D} \boldsymbol{B}(\zeta)|\boldsymbol{J}(\zeta)| d \Omega^{e}
$$

where $\boldsymbol{B}(\zeta)$ and $\boldsymbol{J}(\zeta)$ are the strain matrix and the Jacobian matrix which involve random variable $\zeta$,respectively, $\mathbf{D}$ is the material property matrix.

In conventional SFEM, the element stiffness matrix in the physical space is directly mapped to the parent space in order to perform numerical integration. However, in SIGA framework, three space transformations need to be considered, i.e., an affine mapping from the parent space to the parameter space ( $\mathrm{f}: \tilde{\Omega} \rightarrow \hat{\Omega}$ ); a geometrical mapping from the parameter space to the physical space ( $g: \hat{\Omega} \rightarrow \Omega$ ). Then, these two mappings are combined to constitute space mapping from the parent space into the physical space $(\gamma: f \circ g)$. The mapping processes in the SIGA are illustrated in Figure 4. For a more detailed and thorough discussion of these space concepts, see [12]. 


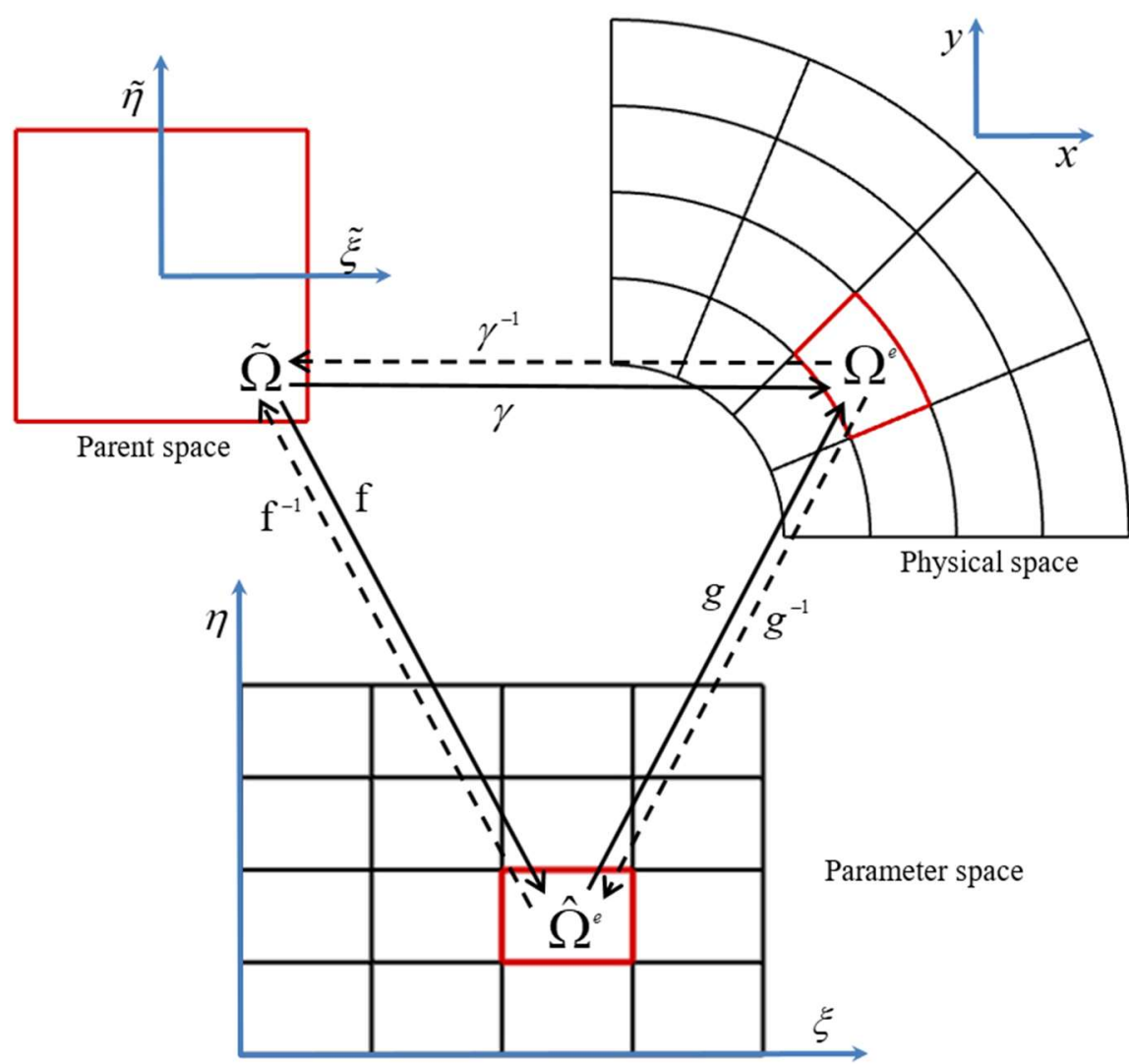

Figure 4: Diagram of mappings in analysis process. Dashed line with arrowhead denotes the inverses of the mappings.

To integrate Equation (33) numerically, the mapping, $\gamma: f \circ g$ was calculated by using the Jacobian determinant including the random variable. Thus, Jacobian determinant for this mapping is expressed by

$$
|\boldsymbol{J}(\zeta)|=\left|\begin{array}{ll}
\frac{\partial \hat{\mathrm{x}}}{\partial \xi} \frac{\partial \xi}{\partial \tilde{\xi}} & \frac{\partial \hat{\mathrm{x}}}{\partial \eta} \frac{\partial \eta}{\partial \tilde{\eta}} \\
\frac{\partial \hat{\mathrm{y}}}{\partial \xi} \frac{\partial \xi}{\partial \tilde{\xi}} & \frac{\partial \hat{\mathrm{y}}}{\partial \eta} \frac{\partial \eta}{\partial \tilde{\eta}}
\end{array}\right|=\left|\begin{array}{ll}
\frac{\partial}{\partial \xi}\left[x+\sum_{i=0}^{n} L_{x_{i}} \Psi(\zeta)\right] \frac{\partial \xi}{\partial \tilde{\xi}} & \frac{\partial}{\partial \eta}\left[x+\sum_{i=0}^{n} L_{x_{i}} \Psi(\zeta)\right] \frac{\partial \eta}{\partial \tilde{\eta}} \\
\frac{\partial}{\partial \xi}\left[y+\sum_{i=0}^{n} L_{y_{i}} \Psi(\zeta)\right] \frac{\partial \xi}{\partial \tilde{\xi}} & \frac{\partial}{\partial \eta}\left[y+\sum_{i=0}^{n} L_{y_{i}} \Psi(\zeta)\right] \frac{\partial \eta}{\partial \tilde{\eta}}
\end{array}\right|
$$

where $\hat{x}$ and $\hat{y}$ are the coordinates involving the random variable $\zeta$ in the physical space (see Equations (25) and (26)). $\xi$ and $\eta$ are the parametric coordinates in the parameter space, and are obtained by the knot vectors and parent element coordinates of the Gauss points, as follows:

$$
\begin{gathered}
\xi=\frac{\left(\left(\xi_{i+1}+\xi_{i}\right)+\tilde{\xi}\left(\xi_{i+1}-\xi_{i}\right)\right)}{2} \\
\eta=\frac{\left(\left(\eta_{i+1}+\eta_{i}\right)+\tilde{\eta}\left(\eta_{i+1}-\eta_{i}\right)\right)}{2}
\end{gathered}
$$


1 where $\tilde{\xi}$ and $\tilde{\eta}$ are the parent element coordinates. In practice, the Equations (35) and (36) 2 involves a mapping which from the parent space to the parameter space $f: \tilde{\Omega} \rightarrow \hat{\Omega}$. 3 Additionally, the mapping from the parameter space to the physical space $g: \hat{\Omega} \rightarrow \Omega$ is 4 calculated from the NURBS basis functions and the control point coordinates. The 5 transformation matrix for mapping $g: \hat{\Omega} \rightarrow \Omega$ is represented by Equations (11), (25) and (26)

$$
\boldsymbol{J}_{g}(\zeta)=\left[\begin{array}{ll}
\frac{\partial \hat{x}}{\partial \xi} & \frac{\partial \hat{x}}{\partial \eta} \\
\frac{\partial \hat{y}}{\partial \xi} & \frac{\partial \hat{y}}{\partial \eta}
\end{array}\right]=\left[\begin{array}{ll}
\sum_{i=1}^{n_{e}} \frac{\partial R_{i}}{\partial \xi}\left[x_{i}+\sum_{j=0}^{n} L_{x_{j}} \Psi(\zeta)\right] & \sum_{i=1}^{n_{e}} \frac{\partial R_{i}}{\partial \eta}\left[x_{i}+\sum_{j=0}^{n} L_{x_{j}} \Psi(\zeta)\right] \\
\sum_{i=1}^{n_{e}} \frac{\partial R_{i}}{\partial \xi}\left[y_{i}+\sum_{j=0}^{n} L_{y_{j}} \Psi(\zeta)\right] & \sum_{i=1}^{n_{e}} \frac{\partial R_{i}}{\partial \eta}\left[y_{i}+\sum_{j=0}^{n} L_{y_{j}} \Psi(\zeta)\right]
\end{array}\right]
$$

where $n_{e}$ is the number of the nonzero NURBS basis function over element $e$, which is equal to $(p+1)^{2}$ in this formulation, and where $p$ is the order of the basis function. The associated 9 Jacobian determinant for this mapping is denoted as $\left|\boldsymbol{J}_{g}(\zeta)\right|$. Thus, the matrix form of Equation (34) is represented by

$$
\boldsymbol{J}(\zeta)=\left[\begin{array}{ll}
\frac{\partial \hat{x}}{\partial \xi} \frac{\partial \xi}{\partial \tilde{\xi}} & \frac{\partial \hat{x}}{\partial \eta} \frac{\partial \eta}{\partial \tilde{\eta}} \\
\frac{\partial \hat{y}}{\partial \xi} \frac{\partial \xi}{\partial \tilde{\xi}} & \frac{\partial \hat{y}}{\partial \eta} \frac{\partial \eta}{\partial \tilde{\eta}}
\end{array}\right]
$$

$$
=\left[\begin{array}{ll}
\sum_{i=1}^{n_{e}} \frac{\partial R_{i}}{\partial \xi}\left[x_{i}+\sum_{j=0}^{n} L_{x_{j}} \Psi(\zeta)\right] \frac{\partial \xi}{\partial \tilde{\xi}} & \sum_{i=1}^{n_{e}} \frac{\partial R_{i}}{\partial \eta}\left[x_{i}+\sum_{j=0}^{n} L_{x_{j}} \Psi(\zeta)\right] \frac{\partial \eta}{\partial \tilde{\eta}} \\
\sum_{i=1}^{n_{e}} \frac{\partial R_{i}}{\partial \xi}\left[y_{i}+\sum_{j=0}^{n} L_{y_{j}} \Psi(\zeta)\right] \frac{\partial \xi}{\partial \tilde{\xi}} & \sum_{i=1}^{n_{e}} \frac{\partial R_{i}}{\partial \eta}\left[y_{i}+\sum_{j=0}^{n} L_{y_{j}} \Psi(\zeta)\right] \frac{\partial \eta}{\partial \tilde{\eta}}
\end{array}\right]
$$

Note that, in this study, uncertainty in shape is considered as the variable random, and represented by introducing PCE into the control points coordinates in the physical space. Therefore, uncertainty representation exists in this mapping process.

In addition, the orthogonal property of PCE is needed to be used in order to perform the formulation. Therefore, the $|\boldsymbol{J}(\zeta)|$ needs to be rewritten as Hermite polynomial form, and obtained by the following procedure: substituting Equations (27) and (28) into Equation (38), solving the Jacobian determinant, and then combining like terms with respect to $\zeta$. Finally, the $|\boldsymbol{J}(\zeta)|$ is expressed as:

$$
|\boldsymbol{J}(\zeta)|=\left|\boldsymbol{J}_{0}\right|+\left|\boldsymbol{J}_{1}\right| \zeta+\ldots+\left|\boldsymbol{J}_{n}\right| \zeta^{n}=\sum_{i=0}^{n}|\boldsymbol{J}| \Psi(\zeta)
$$

where $n$ is the polynomial order. it is equal to 2 , since, in this study, the representation of uncertainty in shape was assumed to follow a normal distribution. 
Moreover, in order to obtain the strain matrix $\boldsymbol{B}(\zeta)$, the derivatives of the basis-functions with respect to the physical coordinates must be calculated. Thus, we first used Equations (35) and (36) to calculate the parametric coordinates with respect to the quadrature points in the parent space. subsequently, the derivatives of the basis-functions with respect to the parameter coordinates were calculated by Equation (9). Finally, the derivatives in the physical space were obtained by applying the chain-rule, as follows:

$$
\begin{aligned}
& \frac{\partial R(\xi, \eta)}{\partial \hat{x}}=\frac{\partial R}{\partial \xi} \frac{\partial \xi}{\partial \hat{x}}+\frac{\partial R}{\partial \eta} \frac{\partial \eta}{\partial \hat{x}} \\
& \frac{\partial R(\xi, \eta)}{\partial \hat{y}}=\frac{\partial R}{\partial \xi} \frac{\partial \xi}{\partial \hat{y}}+\frac{\partial R}{\partial \eta} \frac{\partial \eta}{\partial \hat{y}}
\end{aligned}
$$

where $\partial \xi / \partial \hat{x}, \partial \xi / \partial \hat{y}$ are obtained by calculating the inverse of the mapping from the parameter space to the physical space, and the inverse of this mapping is denoted by $1 /\left|\boldsymbol{J}_{g}(\zeta)\right|$. In this expression, the denominator contains the random variable $\zeta$; therefore, it cannot be directly calculated by applying the PCE. However, $1 /\left|\boldsymbol{J}_{g}(\zeta)\right|$ can be derived approximately by using orthogonally of Hermite polynomials, as follows

$$
\left|\boldsymbol{J}_{g}(\zeta)\right|^{-1}=\sum_{a=0}^{r} \Upsilon_{a} \Psi_{a}(\zeta)
$$

where $\Psi_{a}(\zeta)$ represents the $p$ th-order Hermite polynomials, and $\Upsilon_{a}$ represents the unknown coefficients with respect to the approximate polynomials. We used an algorithm based on the orthogonal property of the Hermite polynomials in order to calculate the unknown coefficients. More details about the algorithm can be found in [10]. After the unknown coefficients were calculated, we were able to determine $1 /\left|\boldsymbol{J}_{g}(\zeta)\right|$, and the applied it to the solution of matrix $\boldsymbol{B}(\zeta)$ in Equation (33). Thus, $\boldsymbol{B}(\zeta)$ is expressed by Equations (40), (41) and (42), as follows:

$$
\boldsymbol{B}(\zeta)=\left[\begin{array}{cc}
\frac{\partial R(\xi, \eta)}{\partial \hat{x}} & 0 \\
0 & \frac{\partial R(\xi, \eta)}{\partial \hat{y}} \\
\frac{\partial R(\xi, \eta)}{\partial \hat{y}} & \frac{\partial R(\xi, \eta)}{\partial \hat{x}}
\end{array}\right]=\left|\boldsymbol{J}_{g}(\zeta)\right|^{-1}\left[\begin{array}{cc}
\frac{\partial R(\xi, \eta)}{\partial \xi}+\frac{\partial R(\xi, \eta)}{\partial \eta} & 0 \\
0 & \frac{\partial R(\xi, \eta)}{\partial \xi}+\frac{\partial R(\xi, \eta)}{\partial \eta} \\
\frac{\partial R(\xi, \eta)}{\partial \xi}+\frac{\partial R(\xi, \eta)}{\partial \eta} & \frac{\partial R(\xi, \eta)}{\partial \xi}+\frac{\partial R(\xi, \eta)}{\partial \eta}
\end{array}\right]
$$

We substituted Equation (42) into Equation (43) in order to rewrite the strain matrix $\boldsymbol{B}(\zeta)$ as PCE form with respect to the random variable $\zeta$ :

$$
\boldsymbol{B}(\zeta)=\frac{1}{\left|\boldsymbol{J}_{g}(\zeta)\right|} \times \boldsymbol{B}_{0}+\frac{1}{\left|\boldsymbol{J}_{g}(\zeta)\right|} \times \boldsymbol{B}_{1} \times \zeta+\cdot \cdot+\frac{1}{\left|\boldsymbol{J}_{g}(\zeta)\right|} \times \boldsymbol{B}_{a} \times \zeta=\frac{1}{\left|\boldsymbol{J}_{g}(\zeta)\right|} \times \sum_{a=0}^{r} \boldsymbol{B}_{a} \zeta^{a}
$$


1 Recall that in the above equations, the strain matrix and Jacobian matrix were all expressed in

2 PCE form. Therefore, we were able to obtain the PCE representation form of Equation (33) as

3 follows:

4

5 Subsequently, we assembled the local stiffness matrix $\boldsymbol{k}_{e}$ into the global stiffness matrix $\mathbf{K}$ and 6 rewrote it in PCE form, as follows:

$$
\mathbf{K}=\sum_{j=0}^{n+r} \mathbf{K} \Psi_{j}(\zeta)
$$

Similarly, the local force vector is expressed as follows:

$$
\boldsymbol{f}_{e}(\zeta)=\int_{\Omega^{e}} R(\xi) \hbar_{e}|J(\zeta)| d \Omega^{e}
$$

where $\hbar_{e}$ is the surface force vector. The PCE form is denoted as:

$$
\boldsymbol{f}_{e}(\zeta)=\sum_{i=0}^{n} \boldsymbol{f}_{e, i} \Psi_{i}(\zeta)
$$

The derivation process of Equation (48) was the same as that of Equation (45); however, due to the applied loads on the single-side, the $f_{e}$ was the obtained by the one-dimensional NURBS basis functions. Then, the global force vector was assembled and represented in PCE form, as follows:

$$
\mathbf{F}=\sum_{i=0}^{n} \mathbf{F} \Psi_{i}(\zeta)
$$

Moreover, the unknown displacement response vector $\mathbf{d}$ in Equation (32) could also be derived approximately by utilising orthogonal property of Hermite polynomials. Thus, we first represented it in PCE form:

$$
\mathbf{d}=\sum_{k=0}^{n+r} \mathbf{d}_{k} \Psi_{k}(\zeta)
$$

Note that the order of PCE for Equation (50) is assumed in accord with that of global stiffness matrix (see Equation (46)), which is needed in order to solve the global stiffness matrix later. The substitution of Equations (46), (49), and (50) into Equation (32), yields:

$$
\sum_{j=0}^{n+r} \mathbf{K} \Psi_{j}(\zeta) \times \sum_{k=0}^{n+r} \mathbf{d}_{k} \Psi_{k}(\zeta)=\sum_{i=0}^{n} \mathbf{F} \Psi_{i}(\zeta)
$$

To solve Equation (51), we utilised the same algorithm used to solve Equation (42). According to the algorithm [10], the orthogonal property of PCE was utilised in order to solve the

$$
\boldsymbol{k}_{e}(\zeta)=\sum_{j=0}^{n+r} \boldsymbol{k}_{e, j} \Psi_{j}(\zeta)
$$


unknown displacement response vector d. First the two-sides of Equation (51) were multiplied by $\Psi_{m}(\zeta) W(\zeta)$ and were then integrated, simultaneously. Thereby, we obtained:

$$
\int_{-\infty}^{+\infty} \sum_{j=0}^{n+r} \mathbf{K} \Psi_{j}(\zeta) \times \sum_{k=0}^{n+r} \mathbf{d}_{k} \Psi_{k}(\zeta) \Psi_{m}(\zeta) W(\zeta) d \zeta=\int_{-\infty}^{+\infty} \sum_{i=0}^{n} \mathbf{F} \Psi_{i}(\zeta) \Psi_{m}(\zeta) W(\zeta) d \zeta
$$

Thus, the inner-product form can be given by

$$
\sum_{j=0}^{n+r} \mathbf{K}\left\langle\Psi_{k}(\zeta) \Psi_{m}(\zeta) \Psi_{j}(\zeta)\right\rangle \sum_{k=0}^{n+r} \mathbf{d}_{k}=\sum_{i=0}^{n} \mathbf{F}\left\langle\Psi_{i}(\zeta) \Psi_{m}(\zeta)\right\rangle
$$

where

$$
\left\langle\Psi_{m}(\zeta) \Psi_{a}(\zeta) \Psi_{i}(\zeta)\right\rangle=\int_{D} \Psi_{m}(\zeta) \Psi_{i}(\zeta) \Psi_{a}(\zeta) W(\zeta) d \zeta
$$

where $\left\langle\Psi_{m}(\zeta)\right\rangle$ is calculated by the Equation (21).

Subsequently, Equation (51) could be rewritten in a form of simultaneous equations by Equation (52), (53), and (54), as follows

$$
\left[\begin{array}{cccc}
\mathbf{K}_{0,0}\langle\cdot\rangle & \mathbf{K}_{0,1}\langle\cdot\rangle & \cdots & \mathbf{K}_{0, n+r}\langle\cdot\rangle \\
\mathbf{K}_{1,0}\langle\cdot\rangle & \mathbf{K}_{1,1}\langle\cdot\rangle & \cdots & \mathbf{K}_{1, n+r}\langle\cdot\rangle \\
\vdots & \vdots & \vdots & \vdots \\
\mathbf{K}_{n+r, 0}\langle\cdot\rangle & \mathbf{K}_{n+r, 1}\langle\cdot\rangle & \cdots & \mathbf{K}_{n+r, n+r}\langle\cdot\rangle
\end{array}\right]\left[\begin{array}{c}
\mathbf{d}_{0} \\
\mathbf{d}_{1} \\
\vdots \\
\mathbf{d}_{n+r}
\end{array}\right]=\left[\begin{array}{c}
\mathbf{F}_{0}\langle\cdot\rangle \\
\mathbf{F}_{1}\langle\cdot\rangle \\
\vdots \\
\mathbf{F}_{n+r}\langle\cdot\rangle
\end{array}\right]
$$

where $\langle\cdot\rangle$ denotes the inner-product with respect to PCE. Finally, the stochastic response of the displacement representing uncertainty in shape was obtained by Equation (55).

In this study, we used the $\mathrm{C}++$ programming language in order to implement the formulation of proposed method. Figure 5 shows a flowchart of program of the SIGA for uncertainty in shape. Clearly, the SIGA program architecture is similar to the IGA program architecture, and the different portions of the program are the those shown in blue in Figure 5. The program begins with the READ INPUT box that read the information containing the geometrical data, stochastic parameter, etc. After the pre-processing steps are completed, the PCE is introduced into element loop and assembly of system, and that plays an important role in these algorithms. Then, the calculation results in regard to displacement response surface and normal probability distribution, etc. are recorded. 


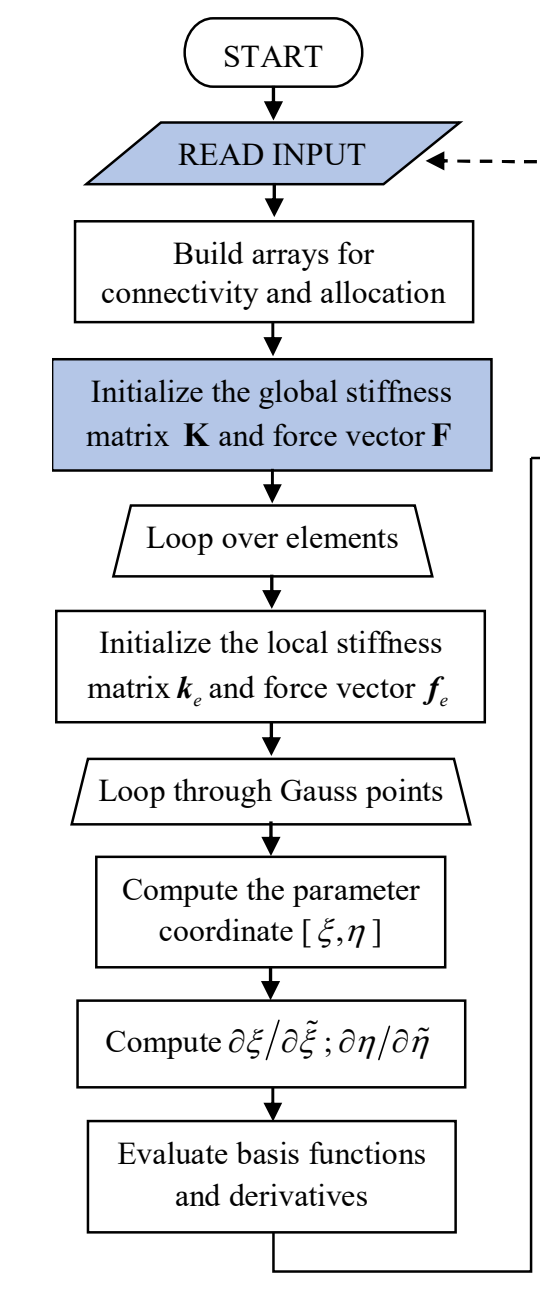

Geometrical data: coordinate of control points, knot vector, etc. Stochastic parameter: mean and standard deviation. Material properties. Boundary conditions.

Figure 5: Flowchart of program for stochastic isogeometric analysis for uncertainty in shape, which applies to single-patch. Routines in blue denote differences from IGA program.

\section{Remark}

(1) In this formulation, the uncertain parameters were only imported into some specific control points in order to represent the uncertainty in shape. These specific control points were determined according to geometrical shape and range that needed to be analysed in the structure model, and these control points had ability to intuitively change shape within this range. Therefore, the uncertainty in shape for structure model could be accurately presented by just importing the uncertain parameters into one or more control points. In this context, this method greatly reduces the computational cost.

(2) The role of PCE is important in the intrusive SIGA formulation procedure. Recalling that the stiffness matrix, force vector and displacement were all rewritten as a representation form of PCE, because we needed to use the orthogonal property of PCE to solve the global stiffness equation.

(3) Note that the algorithms developed so far only apply to Gaussian stochastic fields and processes, and that program of SIGA formulation is implemented based on a single-patch. 
In this section, we apply the stochastic isogeometric analysis framework to a cantilever beam model and a localized corrosion problem. We used chosen examples in order to demonstrate the accuracy and convergence of the proposed method. In all cases, the model problems considered here were linear-elastic problems. Their solutions compare directly to that of MCS used as a reference value. The all calculations are implemented using $\mathrm{C}++$ programming language, and timed on a Windows 10 pro 64bit with Intel(R) CPU E5-2603 v3 @ $1.6 \mathrm{GHz}$ processor (12 CPUs) and 64GB RAM. Additionally, in the figure, the Poisson's ratio and Young's modulus are denoted by $v$ and $E$, respectively.

\subsection{Example 1: quarter-circular cantilever beam}

First, we present a verification example with regard to a quarter-circular cantilever beam. In the setup of problem illustrated in Figure 6, the hash marks on the bottom edge denote the Dirichlet boundary conditions. Additionally, the beam was subjected to a uniform pressure $\left(P_{x}=1 \mathrm{~N} / \mathrm{mm}^{2}\right)$ on its left-edge. The inner and outer radii of the circular beam are denoted as $R_{\text {in }}, R_{\text {out }}$, respectively. The control points used when building the model are plotted in Figure 6(b), while $L_{x}$ and $L_{y}$ denote the deviation length of control point 2 in the x- and y-direction, respectively.

In this two-dimensional example, we assumed that the changes in shape take place in the inner circle. As shown in Figure 6(b), moving any one of the control points 1, 2, and 3 can change the shape of the inner circle. Here, we imported the uncertainty parameters into the coordinate of control point 2 in order to intuitively manipulate the shape of the inner circle. The corresponding standard deviation and mean $L_{x}$ and $L_{y}$ in control point 2 were set to 0.5 and 0 , respectively.

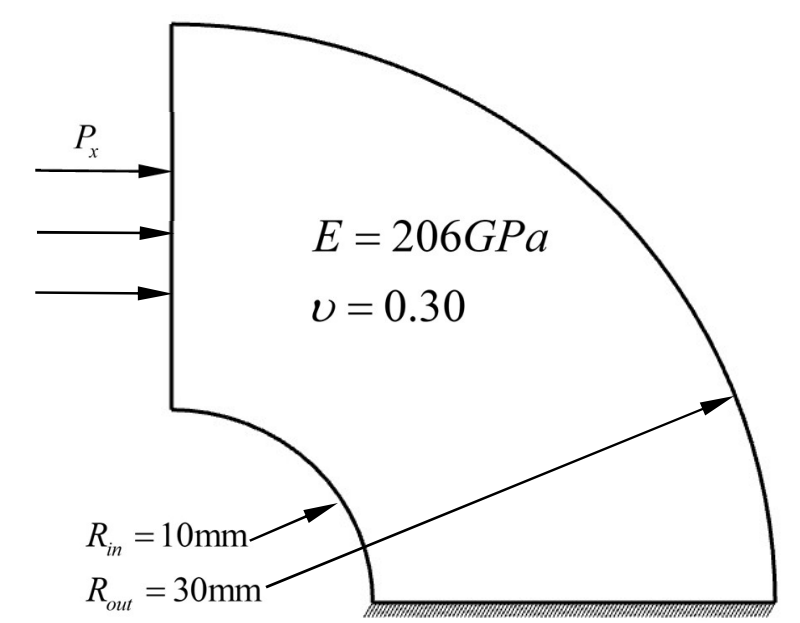

(a) Problem definition for verification example.

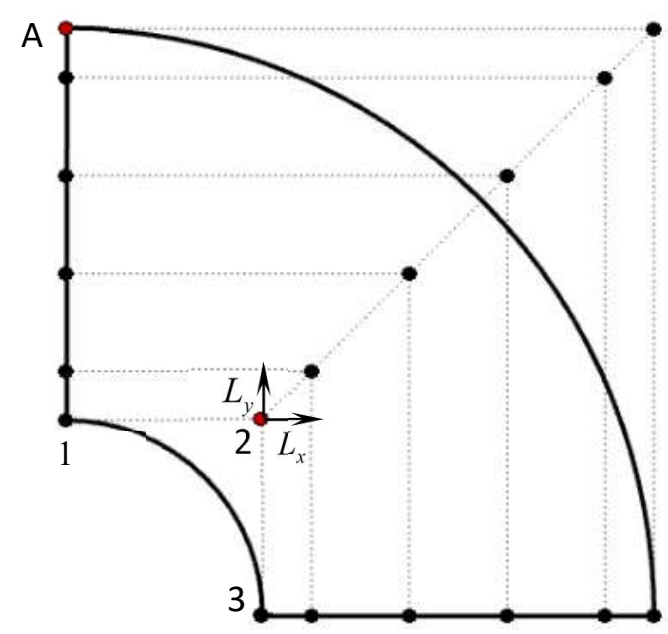

(b) Control points and control net.

Figure 6: Quarter-circular cantilever beam problem 
In this example, we present the analysis results for the displacement response surface in the position of control point $\mathrm{A}$, which is a stress concentration position, and compare them to the analysis results obtained by the MCS and the IGA methods, respectively.

First, in order to demonstrate the accuracy of the analysis results obtained by the proposed method, the results of SIGA and IGA were compared, as shown in Figure 7, which shows that the proposed method had very high precision. The $\mathrm{x}$-axis in Figure 7 represents the stochastic variable $\zeta$, while the y-axis represents the corresponding displacement value. The numerical results of IGA were obtained by modifying coordinate of control point 2 in the $x-$ and $y-$ direction as indicated in Table 1. The response surface of SIGA was computed by using Equation (50). In this example, the order of the Hermite polynomials used to calculate the respond surface was taken as the fourth-order. The polynomial coefficients within the range of 0th to 8th-order are shown in Table 2 and denoted by $a_{i}$. They were derived by Equation (55) in Section 4. Thus, the response surface in this example was obtained by:

$$
\mathrm{d}(\zeta)=1 . \times 10^{-3} \Psi_{0}(\zeta)+9.83 \times 10^{-6} \Psi_{1}(\zeta)+
$$

$$
1.21 \times 10^{-7} \Psi_{2}(\zeta)+2.1 \times 10^{-9} \Psi_{3}(\zeta)-3.31 \times 10^{-10} \Psi_{4}(\zeta)
$$

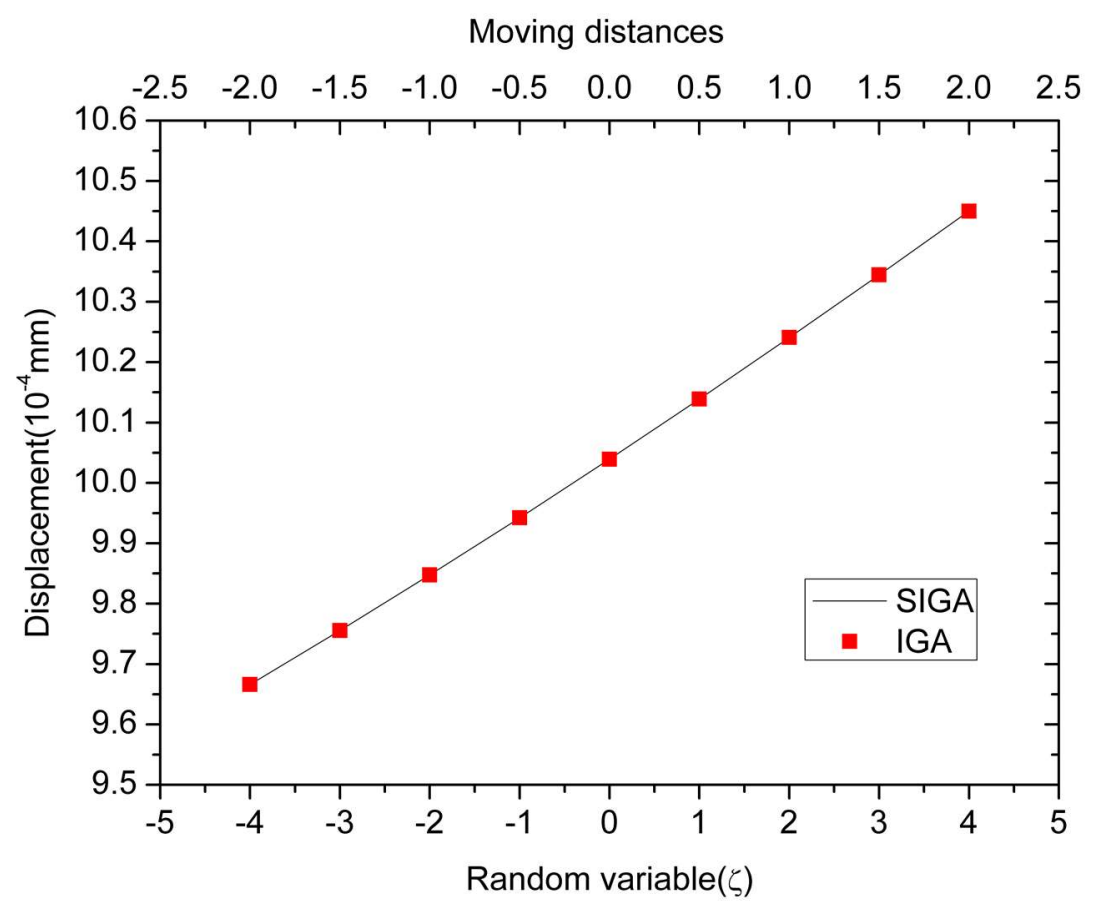

Figure 7: Displacement response obtained by SIGA and numerical results of IGA

In fact, the obtained response surface converged to an exact optimal solution as the order of the polynomial increased. Figure 8, shows error between the analysis results of IGA and SIGA. It can be seen that their errors are very small from the fourth-order of polynomials onwards. Thereby, the demand for precision of analysis results is met and this method is shown to be feasible and accurate. Additionally, the polynomial coefficients of response surface are also 
very small from fourth-order onwards and can be ignored (see Table 2). Based on these considerations, the polynomials in Equation (55) is only computed to the fourth-order.

3 4

6

7

Table 1: Moving distances for control point 2, and corresponding displacement of control point A, indicated in Figure 6(b).

\begin{tabular}{|c|c|c|c|c|c|c|c|c|c|}
\hline $\begin{array}{c}\text { Moving } \\
\text { distances }\end{array}$ & -2 & -1.5 & -1 & -0.5 & 0 & 0.5 & 1 & 1.5 & 2 \\
\hline x-coordinate & 8.00 & 8.50 & 9.00 & 9.50 & 10.00 & 10.5 & 11 & 11.5 & 12 \\
\hline y-coordinate & 8.00 & 8.50 & 9.00 & 9.50 & 10.00 & 10.5 & 11 & 11.5 & 12 \\
\hline $\begin{array}{c}\text { Displacement } \\
\left(10^{-4}\right)\end{array}$ & 9.67 & 9.76 & 9.85 & 9.94 & 10.04 & 10.14 & 10.24 & 10.34 & 10.45 \\
\hline
\end{tabular}

Table 2: Polynomial coefficients of response surface, $\left(a_{i}\right)$.
\begin{tabular}{|c|c|c|c|c|c|c|c|c|c|}
\hline $\begin{array}{c}\text { Order } \\
(i \text { th })\end{array}$ & 0 & 1 & 2 & 3 & 4 & 5 & 6 & 7 & 8 \\
\hline$a_{i}$ & $1.0 \times 10^{-3}$ & $9.8 \times 10^{-6}$ & $1.2 \times 10^{-7}$ & $-2.1 \times 10^{-9}$ & $-3.3 \times 10^{-10}$ & $-2.1 \times 10^{-11}$ & $-2.2 \times 10^{-12}$ & $2.5 \times 10^{-12}$ & $-1.5 \times 10^{-13}$ \\
\hline
\end{tabular}

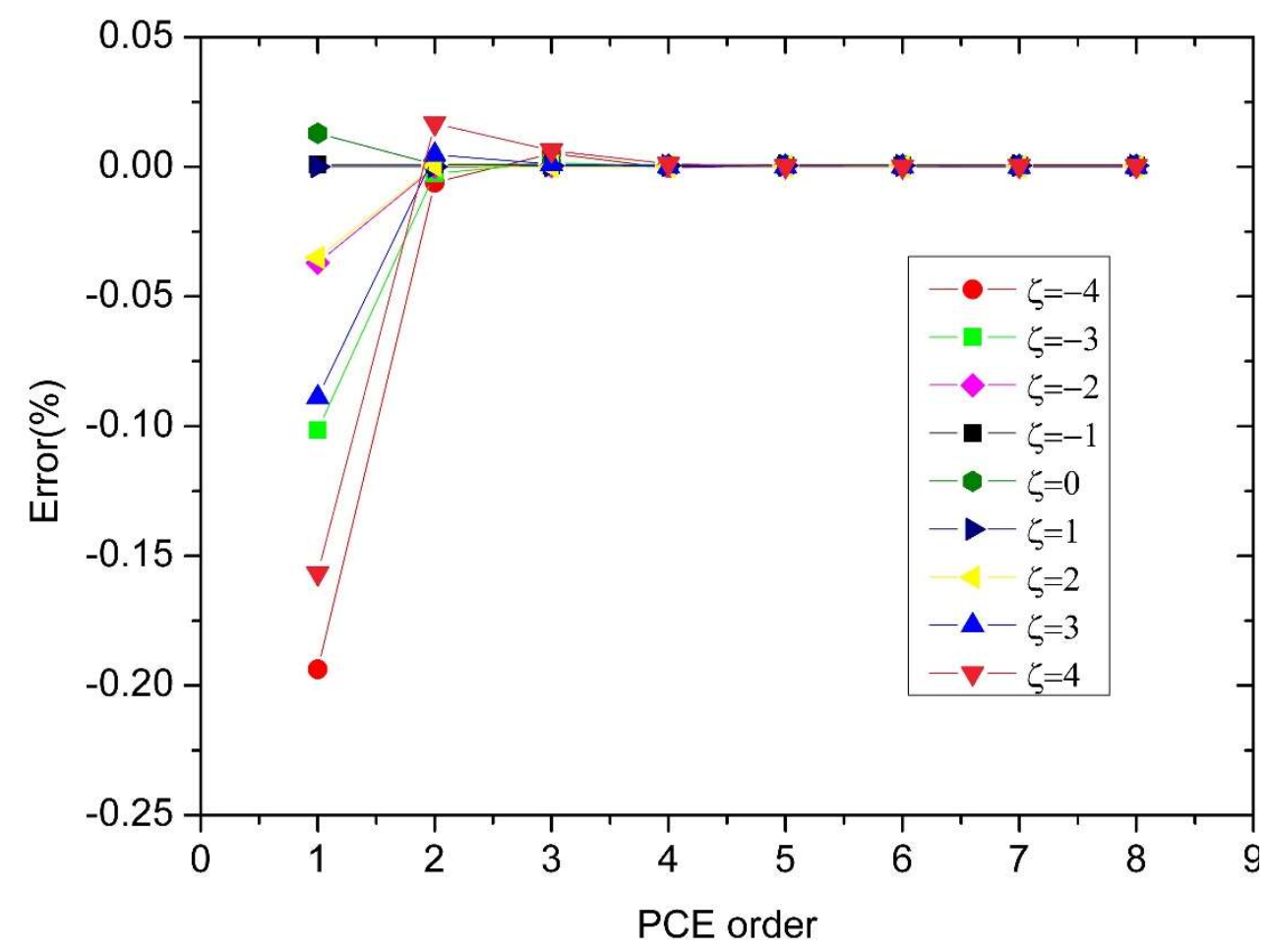

Figure 8: Error measured by numerical results of IGA and SIGA.

Additionally, the statistical characteristics of the proposed method were investigated by comparing with Monte-Carlo simulation. In Figure 9, we present the displacement probability density functions for the control point $\mathrm{A}$ in the $\mathrm{x}$-direction. The reference values (gray clustered column) were obtained by repeatedly performing Monte Carlo simulations with 10,000 model samples and the degrees of freedom in a single sample are 36. The average running time of the program for MSC is 99.196 second. For SIGA, the probability density function of the system was computed by substituting 10,000 random variables $\zeta$ into the Equation (56). The degrees of freedom are 324 and the average running time of the program is the 0.093 second. The 


\begin{tabular}{|c|c|c|c|}
\hline & SIGA & MCS & PCE \\
\hline MEAN $\left(10^{-3}\right)$ & 1.0041 & 1.0039 & 1.0041 \\
\hline STDEV $\left(10^{-6}\right)$ & 9.8175 & 9.8163 & 9.8319 \\
\hline
\end{tabular}

comparison of these results to those obtained by MCS revealed that the probability distribution obtained by SIGA is almost the same as that of MCS, but at a much lower computational cost. Moreover, the mean and standard deviation from these two methods and PCE are listed in Table 3. It can be easily seen that they are almost equal. Note that the mean and standard deviation of PCE are derived by Equations (22) and (23).

Table 3: Mean and standard deviation from SIGA, MCS, and PCE.

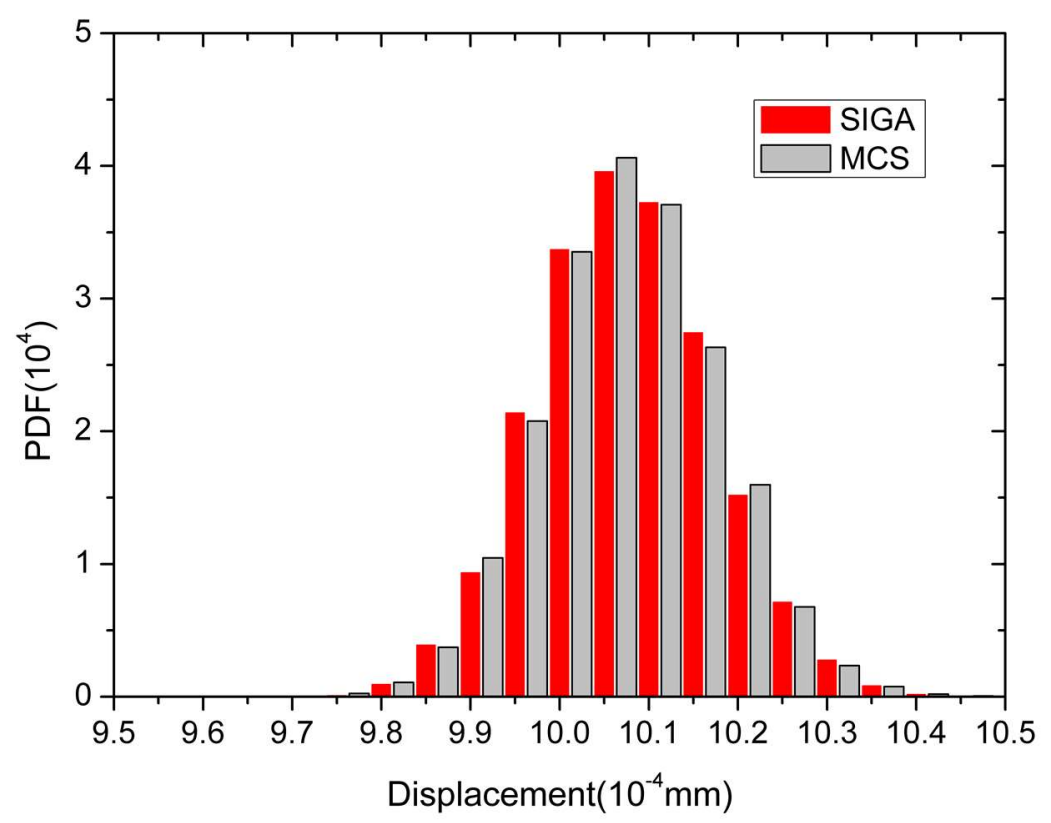

8

9 Figure 9: Displacement probability density functions for the control point $\mathrm{A}$ in the $\mathrm{x}$-direction

\section{Example 2: localized corrosion}

The second example involves a potential application of this method to the problem of uncertainty in shape caused by localized corrosion. The controllability and scalability of proposed method are demonstrated by this example. The relevant setup of the boundary conditions and material properties is illustrated in Figure 10 (a), where the analysis model was subjected to a uniform tensile force $\left(T_{x}=100 \mathrm{~N} / \mathrm{mm}^{2}\right)$ in the $\mathrm{x}$-direction on the right edge. The corresponding control net and control points is shown in Figure 10 (b) where $L_{y}^{m}$ and $L_{y}^{n}$ are the deviation lengths of the control points $m$ and $n$ in the y-direction, respectively. 


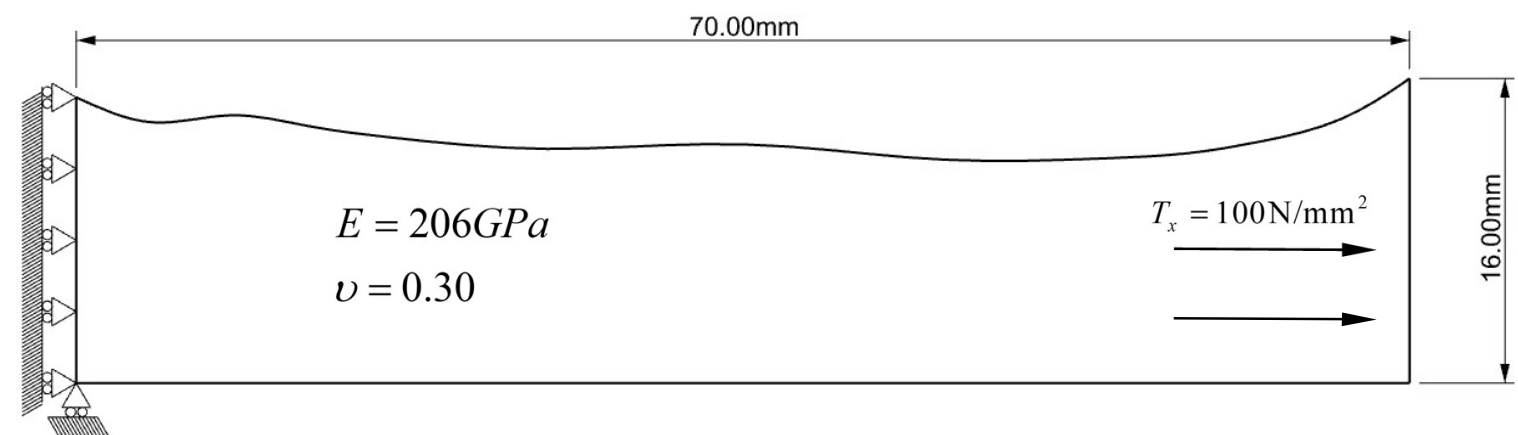

(a) Problem description and data.

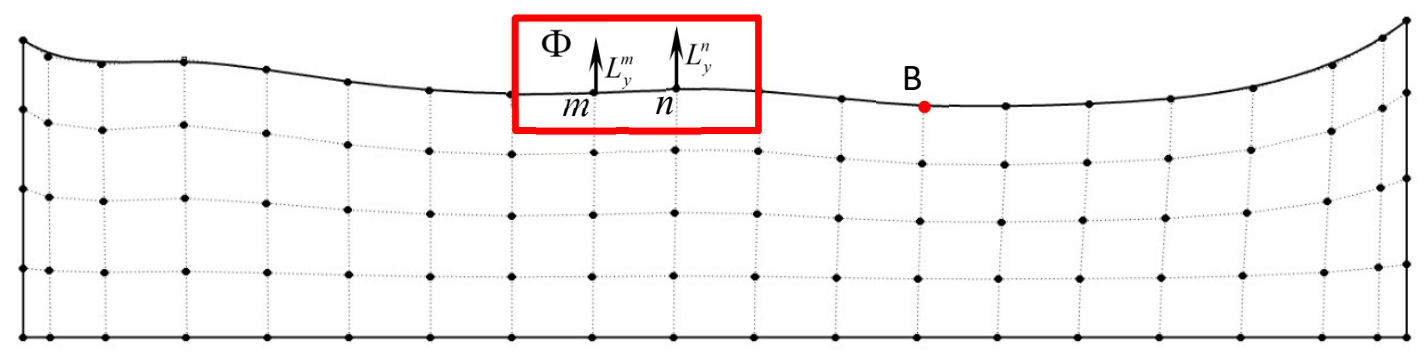

(b) Control net and control points. Rectangular region indicates location where localized corrosion phenomena occurred.

Figure 10: Localized corrosion problem

In this example, we assumed that localized corrosion phenomena appeared on the surface of region $\Phi$ shown in Figure 10 (b). The uncertainty in shape in region $\Phi$ can be represented through the introduction of the uncertainty parameters into the control points $m$ and $n$, since these two control points have the ability to directly change the geometry of this area (see Figure 10 (b)). The corresponding stochastic parameters are shown in Table 4, where acceptable different values are assigned to the mean and standard deviation for the y-coordinate of control points $m$ and $n$, respectively.

Table 4: Stochastic parameters ( $L_{y}^{m}$ and $L_{y}^{n}$ ) for the region $\Phi$ with uncertainty

\begin{tabular}{|c|c|c|}
\hline y-direction & Mean & Standard deviation \\
\hline$L_{y}^{m}($ control point $m)$ & 0 & 0.5 \\
\hline$L_{y}^{n}(\operatorname{control}$ point $n)$ & 0 & 0.6 \\
\hline
\end{tabular}

In what follows, we present the displacement in the $\mathrm{x}$-direction for the position of $\mathrm{B}$ point which is highlighted by red circle shown in Figure 10 (b). They were obtained by using IGA, SIGA methods, respectively. In Table 5, we present the coefficients of displacement response obtained from proposed method (see Section 4). By these coefficients, we computed the displacement response surface shown in Figure 11 and denoted by the solid grey line, while the red-dots denote the reference values obtained by determinate IGA method. by comparing 
the displacements obtained by SIGA and IGA. It can be easily seen that the analysis results of SIGA are in good agreement with the reference values.

Table 5: Coefficients of displacement response, $\left(a_{i}\right)$.

\begin{tabular}{|c|c|c|c|c|c|c|c|c|c|}
\hline Order $(i \mathrm{th})$ & 0 & 1 & 2 & 3 & 4 & 5 & 6 & 7 & 8 \\
\hline$a_{i}$ & $4.5 \times 10^{-2}$ & $-1.8 \times 10^{-3}$ & $2.6 \times 10^{-4}$ & $-2.6 \times 10^{-5}$ & $1.3 \times 10^{-6}$ & $7.5 \times 10^{-8}$ & $-1.6 \times 10^{-9}$ & $-1.8 \times 10^{-8}$ & $2.4 \times 10^{-9}$ \\
\hline
\end{tabular}

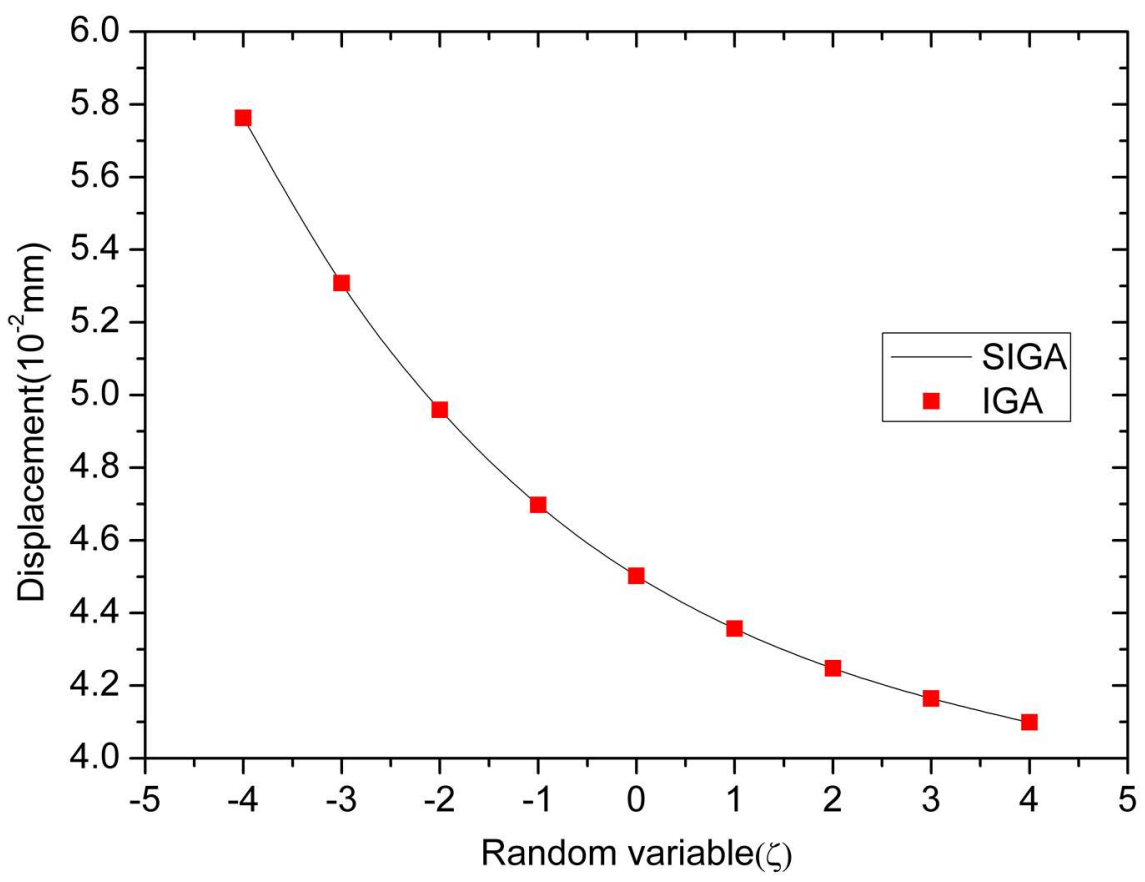

(1)

Furthermore, in Figure 12, we provide the probability density functions of displacement in the $\mathrm{x}$-direction in the position of B point, they were obtained by MCS and SIGA methods, respectively. The results of MCS obtained by running for 10,000 iterations are denoted by the grey-column in Figure12. The degrees of freedom in a single sample are 100. The average running time of the program for MSC is 338.482 second. The results of SIGA were obtained by generating 10,000 random variables $\zeta$ in the same Gaussian random field as MCS, and then introducing the response surface. For the SIGA proposed, the degrees of freedom are 1800 , and the average running time of the program is the 4.816 second. In comparison to MCS, the performance of SIGA was better in terms of computational efforts. Moreover, the mean and standard deviation for MCS, SIGA and PCE are also shown in Figure 12, where it can be seen that their degree of dispersion is almost the same.

This example demonstrates flexibility of the proposed method. In the SFEM analysis framework [8-10], we may need to consider the deformation of the mesh and possible interference between the adjacent nodes, because these factors can affect the accuracy of the analytical results. The SIGA overcame these problems, we could choose the analysis positions 
and range of model freely and flexibly according to the actual situation during the process of analysing uncertainty. Especially, for dealing with complicacy geometry entity, this flexibility and efficiency is even more important.

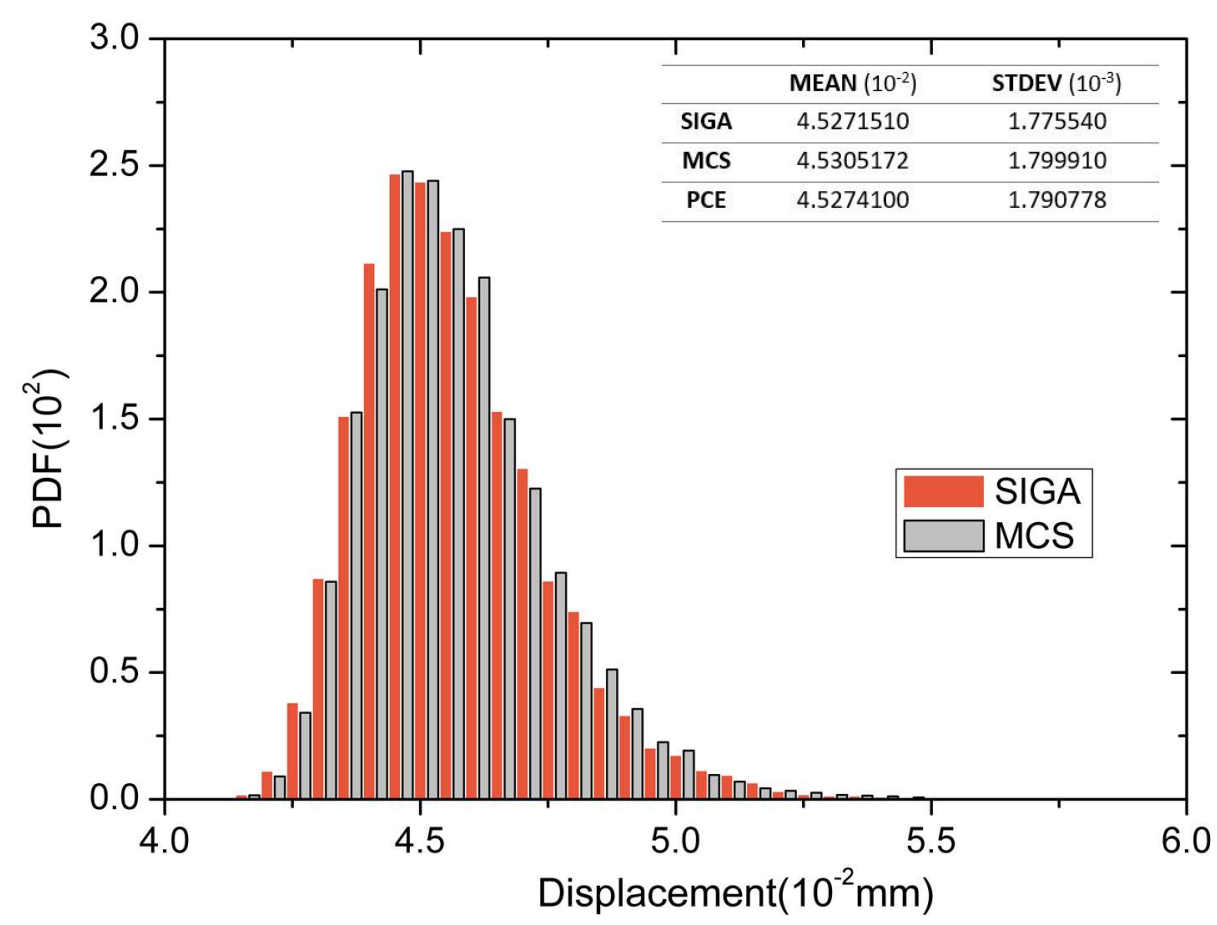

4

Figure 12: Displacement probability density functions for B point position in X-direction. Mean and standard deviation for MCS, SIGA and PCE.

\section{(1)}

This paper presented an innovative numerical method for estimating uncertainty in shape by conjugating the isogoemetric analysis framework and probability theory. In this study, the uncertainty in shape was estimated from a "physically-based" point of view, rather than from the classic "FEA-based" point of view, namely, the uncertainty in shape of structure model was represented by directly introducing stochastic parameters into the control points in the physical space. Additionally, we elaborated on the formulation of SIGA, which is an intrusive formulation procedure. The deterministic isogoemetric analysis framework was rewritten as an uncertainty form based on PCE, and the orthogonal properties of PCE were fully utilised in order to solve the stiffness matrix. Finally, we used the $\mathrm{C}++$ programming language to implement this formulation, and obtained the response surface for the displacement. In order to demonstrate the validity and practical value of the proposed method, the two numerical examples were offered. By these two numerical examples, it could be seen that the numerical solutions from the SIGA were in good agreement with those obtained by IGA and MCS. Moreover, the two examples allowed us to investigate the scalability and applicability of the 
proposed method to very large problems in two- and three-dimensions as well as in parallel implementations.

In future research work, we plan to apply this method to more complex analytical models, especially for the handing of multi-patch and complex CAD boundaries problems, such as multi-patch Coons etc $[25,26]$. Therefore it is indispensable to construct an analysis-suitable parameterization method for SIGA [27]. On the other aspect, we will introduce more stochastic variables into the analytical model in order to represent the more complex stochastic shape.

\section{REFERENCES}

[1] Astill, J., Nosseir, C. J. and Shinozuka, M. Impact loading on structures with random properties. J. Struct.Mech. 1972;1(1): 63-67.

[2] Hurtado J, Barbat AH. Monte Carlo techniques in computational stochastic mechanics. Arch Comput Method Eng 1998; 5(1):3-29. doi:10.1007/Bf02736747

[3] Liu WK, Belytschko T, Mani A. Random field finite elements. Int J Numer Methods Eng 1986; 23(10):1831-1845. doi:10.1002/nme.1620231004

[4] Sudret B, Der Kiureghian A. Stochastic finite element methods and reliability: a state-ofthe-art report (trans: Engineering DoCE) 2000; University of California, Berkeley

[5] Ghanem R, Spanos PD. Stochastic Finite Elements: A Spectral Approach, Springer-Verlag, Berlin (1991), 2nd ed., Dover Publications, NY; 2003.

[6] Blatman G, Sudret B. Sparse polynomial chaos expansions and adaptive stochastic finite elements using a regression approach. Comptes Rendus Mecanique 2008; 336(6):518-523. doi:10. 1016/j.crme.2008.02.013

[7] Honda, R. "Spectral Stochastic Boundary Element Method for Elastic Problems with Geometrical Uncertainty”, Japan Society of Civil Engineers 2004; p111-120. (In Japanese)

[8] Chen X, Kawamura Y, Okada T. A Study on the Method of Structural Analysis with Uncertainty in Shape by Stochastic Finite Element Method (In Japanese). Journal of the Japan Society of Naval Architects and Ocean Engineers 2015; Vol. 22 (2015);187-195

[9] Chen X, Kawamura Y, Okada T. Stochastic finite element method based on response surface methodology considering uncertainty in shape of structures. 13th International Symposium on Practical Design of Ship and Other Floating Structures, Copenhagen, 2016.

[10] Chen X, Kawamura Y, Okada T. Development of structural analysis method with uncertainty in shape to follow non-normal distribution by stochastic finite element method (In Japanese). Transactions of the Japan Society for Computational Engineering and Science 2016; Vol. 2016 (2016) :20160019

[11] Hughes TJR, Cottrell JA, Bazilevs Y. Isogeometric analysis: CAD, finite elements, NURBS, exact geometry and mesh refinement, Comput. Methods Appl. Mech. Engrg 2005; 194 (2005) 4135-4195.

[12] Cottrell JA, Hughes TJR, Bazilevs Y. Isogeometric Analysis Toward integration of CAD and FEA, WILEY; 2009.

[13] Xu G, Mourrain B, Duvigneau R, Galligo A. Parameterization of computational domain in isogeometric analysis: Methods and comparison. Computer Methods in Applied Mechanics and Engineering, 2011, 200(23-24): 2021-2031.

[14] Xu G, Li M, Mourrain B, Rabczuk T, Xu JL, Stephane P.A. Bordas. Constructing IGAsuitable planar parameterization from complex CAD boundary by domain partition and global/local optimization. Computer Methods in Applied Mechanics and Engineering , 2018, $328,175-200$. 

complex objects with topology-consistent volumetric parameterization. Computer-Aided Design, 2017, 91, 1-13.

4 [16] Dimitri R, Fantuzzi N, Tornabene F, Zavarise G. Innovative numerical methods based on 5 SFEM and IGA for computing stress concentrations in isotropic plates with discontinuities, 6 International Journal of Mechanical Sciences 2016; 118 (2016) 166-187.

7 [17] Hien TD, Noh HC. Stochastic isogeometric analysis of free vibration of functionally 8 graded plates considering material randomness. Comput. Methods Appl. Mech. Engrg 2017; 9318 (2017): 845-863.

10 [18] T. D. Hien TD, Lam NN. Investigation into the effect of random load on the variability of response of plate by using monte Carlo simulation. International Journal of Civil Engineering and Technology (IJCIET) 2016; 7(5):169-176.

13 [19] Les Piegl, Wayne Tiller. The NURBS Book, 2nd ed, Springer-Verlag Berlin Heidelberg 14 New York; 1997.

15 [20] Choi SK, Grandhi RV, Canfield RA. Reliability-based Structural Design, Springer, 16 London; 2007.

17 [21] Xiu D, Karniadakis GE. Modelling uncertainty in flow simulations via generalized 18 polynomial chaos. Journal of Computational Physics 2003; 187 (2003) 137-167.

19 [22] Xiu D, Karniadakis GE. The Wiener--Askey Polynomial Chaos for Stochastic Differential 20 Equations. SIAM J. Sci. Comput., 24(2), 619-644.

[23] Chen X, Kawamura Y, Okada T. Development of Stochastic Finite Element Method for Problems with Uncertainty in Shape Following Non-normal Distribution (In Japanese). Conference of JASNAOE 2017; 437-442.

24 [24] Hughes TJR. The Finite Element Method LINEAR: Linear Static and Dynamic Finite 25 Element Analysis, Dover Publications; 2000.

26 [25] Xu G, Mourrain B, Duvigneau R, Galligo A. Constructing analysis-suitable 27 parameterization of computational domain from CAD boundary by variational harmonic method. Journal of Computational Physics , 2013, 252, 275-289

29 [26] Qian Xiaoping, Sigmund Ole, "Isogeometric shape optimization of photonic crystals via 30 Coons patches." Computer Methods in Applied Mechanics and Engineering 200, 2011, no. 25$31 \quad 28,2237-2255$.

32 [27] Xu G, Mourrain B, Duvigneau R, Galligo A. Analysis-suitable volume parameterization 33 of multi-block computational domain in isogeometric applications. Computer-Aided Design, 34 2013, 45(2), 395-404. 